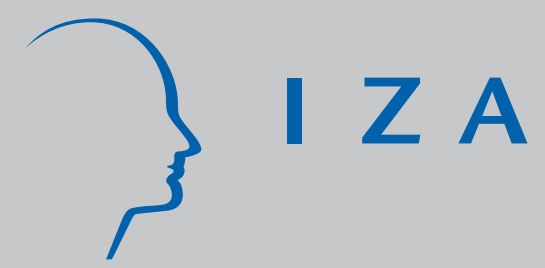

IZA DP No. 9938

Resampling and Bootstrap to Assess the Relevance of Variables: A New Algorithmic Approach with Applications to Entrepreneurship Data

Jose Ignacio Gimenez-Nadal Miguel Lafuente Jose Alberto Molina Jorge Velilla

May 2016 


\title{
Resampling and Bootstrap to Assess the Relevance of Variables: A New Algorithmic Approach with Applications to Entrepreneurship Data
}

\author{
Jose Ignacio Gimenez-Nadal \\ University of Zaragoza and CTUR
}

Miguel Lafuente

University of Zaragoza

\author{
Jose Alberto Molina \\ University of Zaragoza and IZA \\ Jorge Velilla \\ University of Zaragoza
}

Discussion Paper No. 9938

May 2016

IZA

P.O. Box 7240

53072 Bonn

Germany

Phone: +49-228-3894-0

Fax: +49-228-3894-180

E-mail: iza@iza.org

\begin{abstract}
Any opinions expressed here are those of the author(s) and not those of IZA. Research published in this series may include views on policy, but the institute itself takes no institutional policy positions. The IZA research network is committed to the IZA Guiding Principles of Research Integrity.

The Institute for the Study of Labor (IZA) in Bonn is a local and virtual international research center and a place of communication between science, politics and business. IZA is an independent nonprofit organization supported by Deutsche Post Foundation. The center is associated with the University of Bonn and offers a stimulating research environment through its international network, workshops and conferences, data service, project support, research visits and doctoral program. IZA engages in (i) original and internationally competitive research in all fields of labor economics, (ii) development of policy concepts, and (iii) dissemination of research results and concepts to the interested public.
\end{abstract}

IZA Discussion Papers often represent preliminary work and are circulated to encourage discussion. Citation of such a paper should account for its provisional character. A revised version may be available directly from the author. 
IZA Discussion Paper No. 9938

May 2016

\section{ABSTRACT}

\section{Resampling and Bootstrap to Assess the Relevance of Variables: A New Algorithmic Approach with Applications to Entrepreneurship Data*}

In this paper, we propose an algorithmic approach based on resampling and bootstrap techniques to measuring the importance of a variable, or a set of variables, in econometric models. This algorithmic approach allows us to check the real weight of a variable in a model, avoiding the biases of classical tests, and to select the more powerful variables, or more relevant models, in terms of predictability, reducing dimensions. We apply this methodology to the Global Entrepreneurship Monitor data for the year 2014, and find that innovation and new technologies, help others with their business, and that entrepreneurial education at University and the availability of government subsidies, are among the most important predictors for entrepreneurial behavior.

JEL Classification: C21, C52

Keywords: bootstrap, regression, classification, entrepreneurship data

Corresponding author:

Jose Ignacio Gimenez Nadal

Department of Economic Analysis

University of Zaragoza

C/ Gran Via 2

50005 - Zaragoza

Spain

E-mail: ngimenez@unizar.es

\footnotetext{
* This paper has benefited from funding from the Spanish Ministry of Economics (Project ECO201234828). Specifically, ML acknowledges the support from project MTM-2014-53340-P. ML is member of the research group "Modelos Estocásticos", supported by DGA and the European Social Fund.
} 


\section{Introduction}

Statistics is a relatively new field of knowledge that has evolved rapidly in recent decades. Advances in computing, and the recent development of new tools and frameworks such as Bayesian statistics, machine learning, and big data, make Statistics a very dynamic field. But cross-sectional econometric analyses often make use of classical models (linear and logistic regressions) to analyze data and make inferences, and while these models have the advantage of offering easily interpretable results, they rely on strong hypotheses (e.g., homoskedasticity, normality of residuals, and linear incorrelation of regressors) which makes the results and conclusions obtained from the models dependent on the fulfillment of those hypotheses. Certain authors have criticized the conclusions obtained from these methods, as they are not really drawn from the problem (i.e., from the real mechanism of the nature of the problem) but from the correct or incorrect operation of the model (Breiman, 2001b). ${ }^{1}$ Furthermore, the usual measures of the accuracy of models (e.g, BIC, AIC, R-squared, Root MSE, Mean Square Error) may suffer from overfitting (Friedman et Al., 2001). Thus, while the latter approaches are generally accepted in economic analysis, there are sound reasons to propose alternative approaches.

The goal of this paper is to propose an alternative approach based on resampling and bootstrap, to draw conclusions from models where some of the classical hypotheses (e.g., homoskedasticity, normality of residuals, or linear incorrelation of regressors) are not necessarily imposed. Our approach leads us to improved models in terms of accuracy, which may make the results and conclusions drawn from them more reliable in general terms (Breiman, 2001b). To that end, we propose an algorithmic approach that, despite that it is developed for continuous or dichotomous variables, can be extended to other frameworks, such as multiple-categorical variables, by selecting alternative error functions.

The concept of bootstrap was firstly proposed by Efron $(1979,1982)$ as a procedure called "resampling". Since then, many authors have studied the properties of bootstrap and resampling procedures. Horowitz (2003) presents examples of performance of bootstrap econometric techniques. Gonçalves and White (2005) analyze conditions for the consistency of bootstrap estimations and conclude that bootstrap estimates are more accurate than closed-form asymptotic estimates, even in small samples. Bootstrap is also used in two-step econometric models where some regressors are imputed because they are measured with sampling error, and thus second/stage tests based on a covariance matrix are biased (Pagan, 1984; Murphy and Topel, 1985). There are several

\footnotetext{
${ }^{1}$ Some examples of results that depend on the fulfillment of linear and logistic regression hypotheses are the estimated coefficients, their significance, and the accuracy of the model.
} 
books and studies that analyze bootstrap and review its various applications and perspectives (e.g., Vinod, 1993; Jeong and Maddala, 1993; Horowitz, 1995; Davidson and MacKinnon, 2006; and MacKinnon, 2002, 2006). However, less importance has been given in econometrics to problems that are apparently less complicated but are common in social sciences, such as accuracy of regression tests on data that does not satisfy regression hypotheses, and that can be partially addressed with resampling techniques.

Under this framework, we propose an algorithmic procedure where the predictive power of a set of variables is computed, which can then be used to estimate the proportional importance of a variable in an econometric model ${ }^{2}$. In doing so, we eliminate the biases incurred using classical econometric models. Our approach is based on resampling and bootstrap techniques, relies on the honest estimation of the infinite absolute test set error (also called the generalized absolute error in machine learning) and its comparison, and takes into account the risk of overfitting by selecting training and test sets from our samples. This method can hence be seen as an alternative to information criteria and $\mathrm{R}^{2}$ statistics to compare models. We use Global Entrepreneurship Monitor (GEM) National data for the year 2014 to test our methodology. We find that living in a European country is the most important predictor of entrepreneurial behavior. Furthermore, entrepreneurial education, the availability of government subsidies, culture, R\&D transfers, and equity in the access to new technology are the most powerful predictors of entrepreneurial behavior, as their effects are the strongest among all the variables, independently of the significance of $t$-ratios. Furthermore, we repeat the process with the GEM Global Individual Level data for the year 2014, which includes individual level information on entrepreneurial behavior, to test our approach for dichotomous variables. We obtain that helping other entrepreneurs and innovation factors are the main predictors of entrepreneurial activity. In these two applications, our algorithmic approach yields satisfactory results.

Our contribution to the literature is twofold. First, we group some well-known statistical techniques and develop an algorithmic method to compute the predictive power of a variable within a group of variables, and then estimate the strength of its relationship to the outcome using its predictive power. Thus, we redress the fact of assessing the importance of variables through classical and biased tests ( $t$-tests and their significance). The novelty of our approach is not the statistical techniques used, but the fact that such methods are underused in microeconometrics, and we make use of them by aggregation, implementing an algorithmic approach that can be used in academic

\footnotetext{
${ }^{2}$ Friedman (1953) suggested that the predicting power of a model was the key to determine whether relationships between variables were meaningful or not.
} 
settings. Second, we apply this algorithm to two current GEM databases to analyze which variables are best related to entrepreneurial activity.

The rest of the paper is organized as follows. In Section 2, we develop our algorithmic approach. Section 3 presents the data, and in Sections 4 and 5 we apply our methodology to these datasets. Finally, Section 6 presents our main conclusions.

\section{Description of the algorithm}

In this Section, we develop an algorithmic method to measure the importance of independent variables from the point of view of their 'predictive capabilities'. Under this framework, we propose that variables with a high predictive power are "meaningfully" related to the dependent variable, and this significance is more accurate than that of the relationship as measured through classical individual $t$-tests.

The "predictive capability" of a variable depends on the form of the problem. In the case of regression models, we make use of the mean absolute error (m.a.e.) of the prediction (i.e., the average of the absolute differences between real values and their estimations):

$$
\text { m.a.e. }=\frac{\sum_{i=1}^{N}\left|Y_{i}-\hat{Y}_{i}\right|}{N}
$$

where $N$ is the number of individuals, $Y_{i}$ the value of the outcome variable for individual ' $i$ ', and $\hat{Y}_{i}$ its associated predicted value. We choose this measure rather than the mean squared error since its interpretation is clearer (it is measured in the same units as the dependent variable), although the selection should not affect the results qualitatively. ${ }^{3}$ In the case of dichotomous variables, we also make use of the mean absolute error, but comparing the numeric value of the dependent variable, labeled 0 or 1 , with the estimated probability of belonging to the latter class (i.e., each error will be computed with the quantity $P$; the individual does not belong to the real class). ${ }^{4}$

This measure of fitting, in contrast to other measures, such as the $R^{2}$, will not be calculated with the data used to estimate the model because this may lead to overfitting.

\footnotetext{
${ }^{3}$ Mean Square Error (m.s.e.) is generally chosen in statistics because of its differentiable capabilities. However, since we do not make use of these properties, we prefer to choose the mean absolute error.

${ }^{4}$ In the case of a dichotomous variable, we have chosen the mean absolute error rather than the success rate of classification, because for each observation, the former (a number between 0 and 1) gives us more accurate information (sensitivity and sensibility) about the performance of the model than the success-fail result (0 or 1). Also, there may be cases with an unbalanced distribution of the two dichotomous outcomes of interest (i.e., one of the possible outcomes has a high frequency), and thus classical econometric models predicting always the highfrequency outcome could be considered as having a very high predictive power.
} 
This problem appears when we estimate the goodness of fit of a model with the same set of individuals used to train (or fit) this model. When this problem is not taken into account, some noisy information can be taken as good information and then the goodness of fit of the model is overestimated (i.e., the $R^{2}$ strictly increases adding independent variables to the model, although these variables have no relationship with the dependent variable). There are several statistics, widely used, that measure the goodness of fit by penalizing the number of variables. However, these settings still depend on training data (adjusted $R^{2}$ ) and even on the theoretical distribution of the model (e.g., BIC, AIC or AICC). In the same way, the lack of power in goodness-of-fit tests if the alternative direction is not correctly specified has been rigorously proved (Bitel et al. 2006).

In order to avoid this problem, we divide our sample into a group used to fit ('train') the model, a training set (Tr); and another group used to estimate the goodness of fit, a test set $(\mathrm{Te})$. Then, the mean absolute error over the test set takes the form:

$$
\text { m.a.e. }=\frac{\sum_{i \in T e}\left|Y_{i}-\hat{Y}_{i}\right|}{|T e|}
$$

where $|T e|$ indicates the number of individuals in the test set. The estimated m.a.e. (over test set) gives us information on how rightly or wrongly our model predicts new data. Since this method could lead to overfit of the test set, we avoid this by resampling (Friedman et al., 2001; Efron and Tibshirani, 1993).

We compute a bootstrap with 500 iterations on the following algorithm. We first randomly select a bootstrap sample (i.e., we randomly choose with replacement a subsample of the same size as the original sample), which constitutes our training set $\left(\operatorname{Tr}^{(k)}\right.$, for each iteration $k$ ). It is proven that following this rule, we asymptotically get a training set in which approximately $63 \%$ of the individuals in the initial sample appear at least once. The remaining individuals will constitute our test set $\left(T e^{(k)}\right){ }^{5}$ We also randomly select a subset of regressors in each iteration. In our case, we specifically choose the number of introduced features in the model to be on the order of the squared root of the total independent variables, $m_{2} \approx \sqrt{m}$, based on studies for similar problems by Efron and Tibshirani (1993), Amit and Geman (1997) and Ho (1998). We then train our model with the observations of the training set corresponding to the $k$-th iteration:

$$
Y_{i}=\hat{f}\left(X_{1 j}, \ldots, X_{m_{2} j}\right)+\varepsilon_{i}^{(k)}
$$

\footnotetext{
${ }^{5}$ The probability of an observation belonging to the test set is $\left(1-\frac{1}{n}\right)^{n}$, where $n$ is the number of variables. When $n$ goes to $\infty$, this tends to $e^{-1}=0.3678$.
} 
for each $i \in \operatorname{Tr}^{(k)}$, where $X_{1 j}, \ldots, X_{m_{2} j}$ are the features randomly selected and $\varepsilon_{i}^{(k)}$ the unmeasured factors of the corresponding iteration ${ }^{6}$.

For each iteration $k$, once the model is trained, we predict the value of the dependent variable, $\hat{Y}$, for each individual ' $j$ ' in the test set and save those values, as well as the indices of the variables included in the model:

$$
\widehat{Y}_{J}^{(k)}=\hat{f}\left(X_{1 j}, \ldots, X_{m_{2} j}\right)
$$

for each $j \in T e^{(k)}$. Then, the $k$-th iteration finishes and begins the $(k+1)$-th.

Finally, to estimate the overall performance of a particular variable, we average the estimated mean absolute error computed over the corresponding test set for each iteration in which it has participated. The output of our algorithm is then a vector of size of the number of total variables. Each element ' $i$ ' in the vector, $\epsilon\left(X_{i}\right)$, contains the average of the mean absolute error (over test set) of the models in which the $i$-th feature has been added, i.e.:

$$
\text { output }=\left(\epsilon\left(X_{1}\right), \ldots, \epsilon\left(X_{m}\right)\right)
$$

where

$$
\epsilon\left(X_{i}\right)=\frac{\sum_{X_{i} \in \text { Model }_{k}}\left(\text { m.a.e. }\left(\text { Model }_{\mathrm{k}}\right)\right)}{\text { interventions of } X_{i}}
$$

for $i=1, \ldots, m$, and m.a.e.(.) represent the mean absolute error over the test set of the model (equation (2)). Thus, the lower this quantity associated with a variable, the greater the predictive power of the correspondent variable, and thus the greater the importance of such variable, independently from its associated $t$-ratio (and the correspondent $p$-value). Furthermore, we should compare the differences between $\left\{\epsilon\left(X_{k}\right)\right\}_{k \in G}$ and $\left\{\epsilon\left(X_{k}\right)\right\}_{k \notin G}$, to have a precise idea of how important it is to add $X_{k}$ to the model.

We acknowledge that this method allows us to compute the importance of a variable according to its predictive power (i.e., how it helps to predict the dependent variable). However, we do not obtain the sign of the relationship, nor its level of significance based on a test, which is what we pretend to correct due to the limitation of those statistics. While running this algorithm, due to the computational cost of resampling, and the time of fitting each model for big databases, we should be careful when choosing the total number of iterations trying to find equilibrium between this quantity and computation- time trade-off. We also consider the possibility that the

\footnotetext{
${ }^{6}$ In equation (3) it is written for simplicity $Y_{i}$ as a function, although our approaches can be more generally applied to non-functional relationships, as for example, when estimations are based on genetic algorithms or other kinds of stochastic modifications of the more usual statistical learning methods.
} 
number $m_{2}$ of variables participating at each iteration is too high and then we are adding some noisy information to the model. The set of variables included at each iteration of the model does not necessarily provide a good model, and consequently, this measure is not an estimation of the best possible performance. Nevertheless, the quantity will be useful for comparing the power of each variable when it interacts with the whole database information. In sections 4 and 5 , we propose a method to deal with this issue, based on the previously-described algorithm.

It is important to highlight that we do not restrict this analysis to a concrete model (parametric, or not) or algorithm (CART, genetic algorithms...), because the measure of fit of the model, the mean absolute error over the test set, can be extended for every regression problem. An advantage of this algorithm is precisely its capability to be applied to many statistical techniques by changing the regression method or algorithm, despite that these methods could have a stochastic nature. Our methods contain many similarities with the out-of-bag error in random forest (defined originally for tree techniques, Breiman 2001a) or bagging (Breiman, 1996) methods. Nevertheless, out-ofbag estimations are defined for a single model in terms of the bagged model using different loss functions (m.s.e. and rate of failure for regression and classification problems respectively). In addition, since we focus on inference instead of prediction, we choose a more suitable error function, especially in the dichotomous variable setting where the 0-1 loss may be unable to capture some information in problems where the relative frequency of groups is unbalanced. We also formalize, adapt, and combine differently the resampling techniques, playing with stochastic and deterministic variable selection, even across different models, in order to extract information.

Thus, the algorithm allows us to reliably compare some models and choose the best one in terms of its predictability power, but with the aim of making inferences. For example, if we use logistic regressions, we do not need to assume that every hypothesis is true. However, we focus on the degree of prediction of the model and draw conclusions from it, rather than from $p$-values or $t$-ratios that are probably biased because the data may not follow the required hypotheses.

\section{Data and variables}

We first use the Global Entrepreneurship Monitor (GEM) National Level data for the year 2014, in order to analyze which socio-economic characteristics of countries are related to the entrepreneurial activity, which is measured by the TEA index. ${ }^{7}$ This

\footnotetext{
${ }^{7}$ The TEA (Total early-stage Entrepreneurial Activity) index measures the percentage of the working-age population who have begun a new business in the recent 2 and a half years, or intend to do so. This indicator is computed yearly by GEM and is downloadable from http://www.gemconsortium.org/data/sets.
} 
database is annually elaborated by GEM and contains information about nine groups of variables of national, socio-economic characteristics related to entrepreneurship encouragement (a description of those variables can be found in Table 1). This data is based on surveys filled out by 36 experts in each country, where stylized questions are used. For every country, each variable takes the mean average responses of the experts, with values between 1 (totally disagree) and 5 (totally agree). More information can be found in http://www.gemconsortium.org/about/wiki. Since we have data about every single characteristic, and also about each group, or principal factor, of variables (see Table 1), we will repeat the analyses for both cases ${ }^{8}$. We have information on 69 countries: Australia, Austria, Belgium, Canada, Chile, Denmark, Estonia, Finland, France, Germany, Greece, Salvador, Slovakia, Slovenia, Spain, Hungary, Ireland, Italy, Japan, Luxembourg, Mexico, Norway, Netherlands, Poland, Sweden, Switzerland, United Kingdom, United States, Angola, Argentina, Belize, Bolivia, Bosnia, Botswana, Brazil, Burkina-Faso, Cameroon, China, Colombia, Costa Rica, Croatia, Ecuador, Filipinas, Georgia, Guatemala, India, Indonesia, Iran, Jamaica, Kazajistan, Kosovo, Lithuania, Malaysia, Panama, Peru, Puerto Rico, Qatar, Romania, Russia, Singapore, South Africa, Suriname, Thailand, Trinidad and Tobago, Uganda, Uruguay and Vietnam.

Summary statistics of variables and principal factors are shown in Table 1. It is shown how the average TEA index across countries is 13.08, which means that around $13 \%$ of the working age population are entrepreneurs. Furthermore, the standard deviation is 8.15 , indicating a considerable variation across countries. Furthermore, $33.3 \%$ of the countries in the sample belong to the EU, and $42 \%$ to the OECD. The rest of the variables are, in general, between 2 and 3 (in the scale of agreement), with standard deviations rarely greater than 0.5 . The lowest values are found among variables of the group "Entrepreneurial level of education at Primary and Secondary", with a mean value of the factor of 2.09 and a moderate standard deviation of 0.39 . On the other hand, the highest values are reached in the groups "Professional and commercial infrastructures access" (3.02, with a moderate standard deviation of 0.33 ) and "Physical infrastructures and services access" (3.75, with a standard deviation of 0.48 , higher than in the previous case). A recent analysis of the entrepreneurial level of countries using GEM data can be found in Molina and Barrado (2015).

We also use the GEM Global Individual Level of the year 2014 to analyze the individual factors related to the fact of being an entrepreneur, or not. The database is elaborated by GEM on an annual basis and contains information about entrepreneurialrelated characteristics, and is defined at the individual level (microeconomic data). This

${ }^{8}$ GEM NES National Level already defines every factor, as is shown in Table 1. For a given country, each principal factor value is the mean of the single variables' values associated with it. 
data is based on stylized questions elaborated by GEM experts, and filled-in by individuals in each of the analyzed countries. Each question is answered between 1 (totally disagree) and 5 (totally agree). Thus, we are able to analyze entrepreneurship from the point of view of individuals, and macroeconomic factors. More information about this database can be found in http://www.gemconsortium.org/about/wiki.

We now have data on 188,373 individuals, 93,636 of whom belong to an OECD country and 94,738 of whom do not. Summary statistics of the 53 features and the dependent variable are shown in Table 2. We also have information on the country of residence of each individual, information that will be taken into account in the analysis. In order to make the analysis less susceptible to biases, we have redefined variables as dummy, taking value 1 if the answer is an agreement (values 5 as "totally agree" or 4 as "agree"), and value 0 for the remaining categories.

We can see that only $8.1 \%$ of individuals in the OECD are entrepreneurs, in contrast with $16 \%$ in the non-OECD countries. The difference is significant according to the Kruskal-Wallis test. In fact, all characteristics taken into account are significantly different between individuals living in OECD and non-OECD countries, except the perception of the conditions of life. Some of the most meaningful differences can be found in: 1) age (with an average of 43 years in the OECD and 38 in the non-OECD countries); 2) the consideration of the own skills of the entrepreneur (55\% of the individuals in non-OECD countries think they have sufficient skills to be an entrepreneur, in contrast with $44 \%$ of OECD individuals); 3 ) the proportion of individuals who are paid-employed is $54 \%$ in the OECD, vs $39 \%$ in non-OECD countries, which can have important effects on those who are entrepreneurs due to necessity; 4) the desire to become a businessman is present in $66 \%$ of the individuals in non-OECD countries, in contrast with $44 \%$ in the OECD who wish to become an entrepreneur; 5) the consideration of finding good opportunities to become an entrepreneur $(43 \%$ vs $26 \%) ; 6)$ the good social perception of being an entrepreneur (44\% vs $26 \%) ; 7)$ the consideration of an entrepreneurial supporting culture (66\% vs $42 \%)$; and 8 ) the promotion of entrepreneurs through the media ( $64 \%$ vs $41 \%$ ).

\section{An application to continuous variables}

\subsection{First step}

Following the algorithmic approach in Section 2, we consider the TEA variable for the GEM 2014 National Level database, by country, as our dependent variable, and the rest of the variables as regressors. In each iteration $k$, we consider the following linear regression model: 


$$
Y_{i}=\beta_{0}^{(k)}+\sum_{i=1}^{m_{2}} \beta_{i}^{(k)} X_{i}+\varepsilon_{i}^{(k)}, i \in \operatorname{Tr}^{(k)}
$$

with $m_{2}=7$, the number of variables in each iteration, and $\varepsilon_{i}$ being unmeasured errors with a normal centered i.i.d. distribution, obtaining the results shown in Figure $1 .{ }^{9}$ That is, Figure 1 represents, for each exogenous variable (represented on the $\mathrm{X}$ axis) the average value of the mean absolute error (over test sets) associated with the dependent variable (Y axis) considering all iterations in which the corresponding variable appears, which is estimated following the algorithmic approach. That is to say, for every feature on the $\mathrm{X}$ axis, we estimate equation (5) and then represent it on the $\mathrm{Y}$ axis. Thus, we must interpret these results as demonstrating that the smaller the value of the m.a.e. associated with a feature, the better is the model after its inclusion (i.e., the less important the error committed, and the better the prediction).

In Panel A, the process is developed, taking into account all single variables, excluding the principal factors. We find that belonging to the European Union is clearly the most powerful regressor, perhaps indicating the structural characteristics of this group of countries that condition their entrepreneurial level. However, the variable "country" appears to be the less influential regressor, indicating that including country fixed effects, apart from the indicators taken into account in the model, may not contribute in a meaningful way to the accuracy of the models.

Variables D2.4 (“Colleges and Universities provide good and adequate preparation for starting up and growing new firms"), C.1 ("A wide range of government assistance for new and growing firms can be obtained through contact with a single agency") and A.3 ("There are sufficient government subsidies available for new and growing firms") also contribute clearly to model accuracy, in contrast with the rest of the regressors. Thus, our results indicate that entrepreneurial education at University, simplicity in Government assistance, and availability of Government subsidies are among the factors with the greatest impact on the entrepreneurial activity of countries. Variables I.2 (entrepreneurial culture), G1.2 (intra-industry market dynamism), A.4 (funding availability from private investors), G2.5 (restrictions on new firms from established firms), D2.6 (vocational and professional entrepreneurial education) and I.1 (successthrough-effort culture) are, apart from the latter, the independent variables with the highest predictive power, according to our methodology. ${ }^{10}$ On the other hand, the variables D1.3 (entrepreneurial education at primary and secondary levels), G1.1 (goods and services market dynamism), B2.7 (cope with bureaucracy regulation and licensing)

\footnotetext{
${ }^{9} m_{2}$ is the number of variables in each iteration, and we choose it to be around sqrt(total number of variables), as mentioned in the previous section. As we have 56 variables, we take $m_{2}=7$.

${ }^{10}$ GEM's definition of each variable is shown in Table 1.
} 
and E.2 (equity in the access to technology) appear to be the variables with the lowest predictive power.

If we now focus on Panel B (where the analysis is done using the grouped factors, rather than all the single independent variables, and now $m_{2}=3$ ), we can see that, again, belonging to the European Union is by far the most important variable, despite that country fixed effects are among the less important variables. The rest of the results are in line with those found in Panel A, since entrepreneurial education at University (group D.2 in Table 1) and socio-cultural support (Group I) are among the five variables with the higher predictive power, together with R\&D Transfers (Group E) and belonging to the OECD. In contrast, the three variables with the lowest explanatory power regarding the entrepreneurial level of a country are bureaucracy and taxes (Group B.2), country fixed-effects, and Government policies and support (Group B.1).

Our results indicate that entrepreneurship appears to be mainly motivated from vocation, desire, and social norms (Kotsova, 1997; Minniti, 2005; Cooper and Yin, 2005; Arenius and Minniti, 2005; Terjesen and Szerb, 2008; Molina et al., 2015), where government investment and subsidies can be an incentive for individuals to initiate a business and become an entrepreneur (Acs, 1992; Lundstrom and Stevenson, 2002; Amorós et al, 2012; Berrios-Lugo and Espina, 2014) but bureaucracy and taxes restrictions do not discourage them (in contrast with Kotsova, 1997). Furthermore, specific entrepreneurial skills education at University and the professional level appear to also be important determinants for future entrepreneurs (Kotsova, 1997; Minniti and Nardone, 2009; Bosma et al., 2004; Levie and Autio, 2013), together with concrete issues at early stages of education, where it is important to promote creativity and selfsufficiency (in line with the notion of Kyrö, 2015, that entrepreneurial education is a new form of pedagogy).

\subsection{Step by step selection.}

We now repeat the procedure but selecting only one variable in each iteration, and increasing the number of iterations to 2,000. Our objective is to check, in a simple linear regression model, which variable has the greatest predictive power. Figure 2 shows the results, where the $\mathrm{X}$ axis shows the variables, and the $\mathrm{Y}$ axis the average of the m.a.e. associated with each variable. However, we will select in each of the steps (each panel of this figure) only the most predictive feature, which will be included in the next fitted model. The process ends when the average m.a.e. of the best variable included is worse than the previous one, indicating that its inclusion does not suppose any improvement in the model. We can see in Figure 2, Panel A, that the dummy EU is the first variable to enter into the model. It is important to note that the mean average error in the model 
whose only regressor is belonging to the $\mathrm{EU}$ is 5.35, almost equal to the mean average error of the 'best' variable of the previous subsection, which leads us to consider that most of the information in the database is noisy with respect to the TEA index. What we propose here is to discover more information about the relationships between variables as a modification of our algorithm based on forward stepwise variable selection.

We continue to estimate the mean absolute error over the test set in the same way via bootstrap samples, but taking the EU variable (the one with the lowest error) and one other. For this second variable, a loop will be in charge to set an equal number of participations in the model for every regressor.

Mean prediction errors over test sets are shown in Figure 2, Panel B. We see how the most predictive model is the one with EU and the R\&D level of transference as regressors of the TEA index, with an error of less than 5.1, which supposes an increment of predictive power with respect to the previous case.

Repeating analogously and including a third variable, we find that the best feature would be the entrepreneurial level of education at vocational, professional, College, and University (Figure 2, Panel C), giving us a new estimation of the mean absolute error of prediction of 5.00. Finally, if we attempt to add another variable following the same approach, we find that the best variable would be the financial environment related to entrepreneurship (Figure 2, Panel D). However, its inclusion supposes a new mean prediction error of 5.06, which is worse in comparison to the previous model.

Thus, we find that the final model for the TEA index, taking into account overfitting, and considering the principal factors from the GEM database as regressors, is the one including the dummy variable for belonging to the EU, the R\&D transfers (Group E), and the level of vocational, professional, College, and University entrepreneurial education (Group D.2). This result should be taken into account, since adding more variables could result in adding noisy information. Since econometric models have the goal of providing the most adequate conclusions, in terms of the economic theory, predictability should play a primary role because it gives researchers an idea of how accurate their conclusions are, relative to the data.

Figure 3 shows the results of the analysis when all variables are included rather than the principal components. We find that the variables that should be included in the model are, in order of appearance in the algorithm: belong to the EU (Panel A), A.3 (government subsidies for new and growing firms, Panel B), D2.4 (entrepreneurial preparation in Colleges and universities, Panel C), D1.1 (creativity, self-sufficiency and initiative in primary and secondary education, Panel D) and E.2 (new and established firms have as much access to new research and technology, Panel E); including I.3 (national culture encourages entrepreneurial risk-taking, Panel F) should be the 
following choice, but it increases the mean prediction error, and we conclude that the information contained in this variable is of little use in the presence of the previouslymentioned variables.

In order to have reliable results, we usually choose a large number of iterations regarding the size of the database, due to the stochastic nature of our algorithm, and thus it is clear that the step selection should always be performed forwards, due to the notable computational effort of fitting models in high dimensions. This problem arises, for example, in the next section for individual data, where a backward approach would be nearly useless.

\section{An application to dichotomous variables}

Although in this section, the dependent variable is categorical (dichotomous), the same main ideas apply. As previously described, our methods not only allow us to find the variables with the strongest relationships in terms of their predictive power, but also the measure of goodness of fit allows us to compare models. We will compare the performance of a logistic regression model and a linear discriminant analysis (LDA) model in predicting the probability of the contribution to the TEA index outcome, making use of the GEM 2014 Global Individual database.

We predict the probability of the dependent variable, taking the value 1 (i.e., the individual is an entrepreneur, 0 otherwise) and, for each feature and each iteration $k$ in which it participates, analogously as in subsection 2.1., we keep the mean of the absolute value of the difference between the real values of $Y$ and the predictions, $\hat{Y}$, as follows:

$$
\text { m.a.e. }\left(\operatorname{model}_{k}\right)=\operatorname{mean}\left(\left|Y_{j}^{k}-\hat{Y}_{j}^{(k)}\right|\right)
$$

for each $j \in T e^{(k)}$.

Then, we take for each variable the mean of m.a.e. $\left(\operatorname{model}_{\mathrm{k}}\right)$ for all the iterations in which it participates (Equation 5), obtaining an honest estimation of the mean absolute prediction error of the correspondent variable.

In the logistic regression model, for an individual ' $i$ ', we assume that the dependent variable $Y_{i}$ follows a Bernoulli distribution with parameter $p_{i}$, where we must estimate the coefficients of the following equation:

$$
\operatorname{logit}\left(Y_{i}\right)=\ln \frac{p_{i}}{1-p_{i}}=\beta_{0}+\sum_{k=1}^{K} \beta_{k} X_{i k}+\varepsilon_{i}
$$


where $\left\{X_{k}\right\}_{k=1}^{K}$ are the regressors and $\varepsilon_{i}$ is the error term. This expression gives us a final estimation of the probability of belonging to the class labeled as 1 , as follows:

$$
p_{i}=\frac{e^{\beta_{0}+\sum_{k=1}^{K} \beta_{k} X_{i k}}}{1+e^{\beta_{0}+\sum_{k=1}^{K} \beta_{k} X_{i k}}}
$$

On the other hand, LDA, sometimes called the Fisher Discriminant Analysis, assumes the distribution function of each target group to be multivariate normal. Linear discriminant analysis also assumes the covariance matrices to be equal in every group (homoskedasticity), in contrast with Quadratic Discriminant Analysis. In this way, the density of each group $k$ (in our case $k=\{0,1\}$ ), is supposed to be bell-shaped

$$
f_{k}(x)=\frac{1}{(2 \pi)^{p / 2}|\Sigma|^{1 / 2}} \exp \left(-\frac{1}{2}\left(x-\mu_{k}\right)^{T} \Sigma^{-1}\left(x-\mu_{k}\right)\right)
$$

where $p$ is the number of regressors, ${ }^{\mathrm{T}}$ is the transposition operator, $\mu_{k}$ is the vector of means of dimension $p$ for the $k$-th group, and $\sum$ the covariance matrix, which is equal across every group, as pointed out above. Under these assumptions, Bayes' Theorem gives us the estimated probabilities of belonging to a group $k$ for a particular individual with attributes $x$ as follows

$$
p_{k}(x)=\frac{\pi_{k^{*}} \frac{1}{(2 \pi)^{p / 2}|\Sigma|^{1 / 2}} \exp \left(-\frac{1}{2}\left(x-\mu_{k}\right)^{T} \Sigma^{-1}\left(x-\mu_{k}\right)\right)}{\sum_{l=0}^{1} \pi_{l^{*}} \frac{1}{(2 \pi)^{p / 2}|\Sigma|^{1 / 2}} \exp \left(-\frac{1}{2}\left(x-\mu_{l}\right)^{T} \Sigma^{-1}\left(x-\mu_{l}\right)\right)},
$$

with $\pi_{k}$ being the prior probability of each group. Thus, in order to fit this model, we need to know the a priori probabilities, the mean vectors, and the covariance matrix for each group. As is usual in this setting, mean vectors and the covariance matrix can be the estimated by maximum likelihood, while prior probabilities can be estimated with the relative frequency of each group in the trained set.

Finally, we remark that this technique is called linear because the decision rule to classify an individual in any particular class has a linear form. Performing some algebra, we arrive at the following discriminant function or decision rule:

$$
\delta_{k}(x)=x^{T} \Sigma^{-1} \mu_{k}-\frac{1}{2} \mu_{k}^{T} \Sigma^{-1} \mu_{k}+\ln \left(\pi_{k}\right),
$$

and consequently, we will assign the observation with features $x$ to the class in which $\delta_{\mathrm{k}}(x)$ is larger. As a consequence of this result, LDA analysis assigns a single coefficient to every variable, with these coefficients being the final weight in the decision rule, and describing the effect of the regressors by the response.

Figure 4, Panel A shows the result of applying our algorithm to a logistic regression model, making use of the GEM 2014 Individual data with 500 iterations. The interpretation of this figure is analogous to the interpretation of Figure 1. For each 
variable (on the $\mathrm{X}$ axis), we plot the average of the m.a.e. (Y axis), considering all the iterations in which the characteristic appears, and then, the smaller the mean error, the better the prediction and the more important is the corresponding feature. We find that the most important variables (and then the variables with the greatest effect according to their predictive power) are searching for a new market (index 23), having recently helped other entrepreneurs (index 9), can offer a new product and new technologies (indexes 10 and 11, respectively), and being a businessman or self-employed (indices 118 and 14). In particular, we find strong and unbiased empirical evidence on the importance of oral and meeting transmission as a determinant factor of entrepreneurial activity (in line with Holcomb et al's., 2009 entrepreneurial learning by seeing, and Blumberg and Pfann's, 2015 parent's transmission). We also find evidence on the importance of innovation as a determinant of becoming an entrepreneur (Schumpeter, 1934; Gilbert, McDougall and Audrestch, 2006).

With the same number of iterations, we repeat the analysis, applying the algorithm to an LDA model. Results are shown in Panel B of Figure 4. Although the three former variables in the previous paragraph appear again among the most important variables, we find that the order is different, in that "have helped others to entrepreneur" now appears to have a stronger effect than "being self-employed". Furthermore, "being a businessman" is not among the five most important variables (it is the sixth), in favor of the variable "new market". The intuitive explanation behind these results is essentially the same as in the previous case.

In view of our results, we remark that variables that tend to decrease the prediction error are the same in general for both models. The fact of their being important variables, regardless of the initial hypothesis, gives us strong evidence of the correctness of our conclusions.

Despite the remarked similarities, a significant difference between the logistic regression and the LDA models applied to our data is the scale of the error estimations. The minimum values for the former are around 0.09 , while minimum values for the latter are around 0.06. Then, given these results, it seems that for this particular case the LDA model plays a more important role than the logit model.

\subsection{Step by step selection and some remarks}

Econometric models in the field of micro-econometrics for dichotomous variables tend to include many features of regressors, and the problem of overfitted models may also have a strong presence in these analyses. In order to select the most important variables and avoid this problem, we apply again a step-by-step forward selection from the GEM 
2014 individual data. As we noted above, due to the size of the database, a backward selection would take too much computing time. Thus, we analyze the prediction error for the first steps of a forward stepwise selection with 10 iterations for each step and each variable.

Figure 5, Panel A shows results for the logistic regression model, and Panel B for the LDA model. We represent on the $\mathrm{X}$ axis the best predictive features, ordered by importance, and on the $\mathrm{Y}$ axis the associated average of the m.a.e. over the test set of the model that includes the corresponding and the previous features. Then, the smaller the associated average m.a.e. associated with a variable, the better the model, including this variable and all previous ones.

We find that, for the second case, the ten 'best' variables are, in order: "have helped other entrepreneurs", "have new technologies available", "belong to a new market sector", "being a businessman", "age", "being an active unemployed", "being satisfied with the current job", "living in Israel", "consider to have opportunities to entrepreneur", and "living in China". Among our estimations, we get the minimum for the generalization absolute error of predicted probabilities of around 0.046 with the six first variables. Adding more variables does not improve the model in terms of the prediction error, indicating that these six offer us the best performance. Furthermore, independently of the possible existence of a statistically significant relationship between the rest of potential regressors and the output variable, its inclusion in the model does not add useful information. Multiple reasons can originate this phenomenon, with this behavior for the $p$-values being very common, especially in large databases. For example, the existence of co-linearity with the six included regressors, possibly combined with a fit of noise when dealing with the whole database. This last reason is often related to inadequate hypothesis, in practice.

We can also discern a significant difference in the error term when we include the fourth variable, but only minor differences with the addition of the fifth and sixth. Thus, its inclusion may slightly complement the information offered by the four previously added variables. In any case, we remark that after the sixth variable the estimation of the mean absolute error over the test set tends to be flat for an increasing number of variables. In fact, this is a typical phenomenon in statistical learning methods when assessing accuracy. We can observe a characteristic U-shape for the estimated test error, which in this case, due to the large number of individuals in the database, is attenuated and appears flat. This behavior contrasts with the estimated error in the trained set, which is always decreasing when the flexibility of the model, in this particular case the number of variables, increases. The relation between the behaviors of the two kinds of 
error is part of a wider issue present in every problem of supervised learning, and is called the bias-variance tradeoff.

These results indicate that, apart from the variables mentioned in the previous paragraphs being important determinants of entrepreneurial activity, age should always be taken into account in entrepreneurial empirical models (Davidsson, 1989; Blanchflower, 2000; Schott and Bagger, 2004; Kelley,2009). Furthermore, the fact of being an active unemployed should also be considered. This is directly related to entrepreneurship due to necessity: individuals who are unemployed and have no income may decide to start their own business as an alternative.

If we now focus on the logit model results (Panel B, Figure 5), we find that the ten variables that the forward stepwise algorithm has included in the model are, in order: "have helped other entrepreneurs", "belong to a new market sector", "age", "being a businessman", "having ended a business recently", "have new technologies available", and "living in Botswana", "Colombia", "Malawi", and "Vietnam" (all of these are not developed countries). Although the list of variables included has meaningfully changed, those in the first part, i.e., the ones that should really be taken into account before adding useless information to the model, have changed hardly at all. Note that now the optimum number of variables to add should be five (since the inclusion of the sixth increases the error estimation), where the first four features are among the five best in the LDA analysis. It should also be taken into account that the scale of the error term is again higher in the case of the logit model than in the LDA model. Some details about the final chosen logit model can be found in Table 3 .

Again, for the logistic regression model, the estimated mean absolute error becomes stable after the inclusion of the first five variables. Furthermore, Panel A shows that the following variables to be added to the model are the dummy variables that define the country of residence of individuals. This confirms the results of Figure 4: once these groups of variables are taken into account, the addition of more variables may be just adding noisy information to the model, independently of it being a logistic regression or a LDA. Then, once the variables "have helped others to become entrepreneurs", "belong to a new market sector", "age", "being a businessman", "having ended a business recently", "being an active unemployed" and "have new technologies available" are included in the model, the inclusion of other variables overfits the model and conclusions drawn from it may then be biased. So, we conclude that, although a bidimensional analysis could show strong relationships between other variables and the contribution to the TEA, its contribution to the prediction weakens in the presence of the cited variables. 
As final remarks, we see the effect of the variable "can offer a new product". In Figure 4, we have found this to be one of the most important regressors. However, it does not appear as a chosen variable for the model as a result of the forward selection (in Figure 5). This is a perfect example to illustrate why it is important to take this second phase of stepwise selection of variables. According to Figure 4, this innovation variable is one of the most notable regressors. However, from Figure 5, we can conclude that the information about the TEA index that this variable has is previously contained in other variables, which are already in the model (because the relationship of the combined variables chosen with the TEA is higher). This example is intuitive, because there is a significant difference between the error terms of the 'best' variables and the rest. However, in other databases, where this difference is not as pronounced as here, it may happen that a variable with a high predictive global power, but which is correlated with some others of greater importance, cannot easily be found. An alternative could be to run again our algorithm with some variables fixed (those that we have decided to include following the forward stepwise procedure, prior to a high 'decreasing jump' in the error terms), and others randomly selected. Then, we could check whether this 'decreasing jump' in the error term could also be attributed to variables other than the ones already included in the model.

Another variable with a clarifying behavior for the suitability of our techniques is that of "belong to a new market". In Table 3, we can see that the associated $p$-value for this mentioned variable is around 0.75 , which in practice is usually viewed as a very high value, perhaps indicating a weak effect on the model. In addition, in a simple logit model with the "belong to a new market" variable being the only feature among the regressors, its associated p-value rises to 0.77 and the estimated coefficient yields a value of 21.93. We also know that the best possible performance for a logistic regression model without any regressor can be achieved by assigning the majority class for every individual, giving us a rate of failure in classification of $12.2 \%$. However, including in the analysis the new market variable, which is supposed not to be a good predictor according to its $p$-value, via resampling we obtain an estimated rate of failure in classification of $8.447 \%$, which supposes a large improvement in the reduction of the considered error. This kind of error decreases to $4.239 \%$ when every selected variable in the stepwise selection is added to the model.

\section{Conclusions}

This paper proposes a way to analyze the predictive power of regressors in crosssectional data models. We make use of resampling and bootstrap techniques to estimate the importance of variables from their predictive power, based on the mean absolute 
error. We quantify the role of a regressor by the difference between the real response value and the predicted value when this regressor is added. Against this background, the risk of overfitting is mitigated and the biases of the classical significance tests with strong hypotheses (that are hardly ever followed by the data) are also avoided. Thus, we implement an algorithm with some modifications which, cleverly combined, allows us to alternatively and more accurately analyze the predictive power of a model (or a set of models) and the real weight of the variables in those models. We also apply this procedure to two GEM 2014 databases (NES National and Global Individual Level) to analyze which macro- and micro-economic variables are more helpful in predicting entrepreneurial activity. We apply the algorithm to linear regression, logistic regression and linear discriminant analysis models.

Our work contributes to the literature by offering an algorithmic approach to check the importance of variables, and also to reduce dimensionality by identifying the best and worst variables in terms of accuracy. In doing so, we bootstrap the estimations (the training of the model) and compute the test-set mean absolute error, randomly selecting the regressors in each iteration. Furthermore, we compare results with a one-by-one selection of variables, following the same algorithmic procedure, in order to detect multicollinearity and avoid overfitted models. The application of the developed tools to GEM databases shows, first, the strength of innovation and research, and of entrepreneurial education (at the professional, vocational, College, and University stages) as factors determining entrepreneurship. Second, taxes and bureaucracy appear not to be a burden for entrepreneurs, which agrees with the Molina and Velilla (2015) hypothesis that entrepreneurship is a vocational activity. Subsidies and Government programs supporting entrepreneurship appear not to be effective in overall terms, although certain characteristics of them, such as ease of access, may be effective. Third, at the individual level, innovation factors, and having contact with other entrepreneurs in the past (e.g., knowing other entrepreneurs, helping other entrepreneurs in their work activities) are among the most important determinants for the participation in the TEA index.

Thus, our results should be taken into consideration in order to develop future research about entrepreneurship. Furthermore, European politicians are currently using entrepreneurship as a way to improve economic growth and reverse the negative consequences of the recent economic crisis. In doing so, they are investing large amounts of money in promoting entrepreneurial activity. However, as argued in Naudé (2016), it is not clear whether the effect of entrepreneurship on economic growth is significant, or not. Furthermore, there is evidence suggesting that those policies that pretend to provide incentives to entrepreneurship are not particularly effective and efficient (Nagler and Naudé, 2014; Karlan and Valdivia, 2011). Because of this, policy 
makers should take into account these results as a guide to a more efficient promotion of entrepreneurship.

\section{REFERENCES}

Acs, Z. (1992). "Small business economics: A global perspective," Challenge 35, 38-44.

Amit, Y. and D. Geman (1997). "Shape quantization and recognition with randomized trees," Neural Computation 9, 1545-1588.

Amorós, J.E., Etchebarne, S. and C. Felzensztein (2012). "International entrepreneurship in Latin America: Development Challenges," ESIC Market Economics and Business Journal 43, 497-512.

Arenius, P. and M. Minniti (2005). "Perceptual variables and nascent entrepreneurship," Small Business Economics 24, 233-247.

Berrios-Lugo, J.E. and M.I. Espina (2014). "Determinant factos for the development of entrepreneurial activity: A correlational study," ESIC Market 147.

P.J. Bickel, Y.Ritov and T.M. Stoker (2006). "Tailor-Made Tests for Goodness of Fit to Semiparametric Hypotheses", The Annals of Statistics, Vol. 34, No. 2 (Apr., 2006), pp. 721-741.

Blanchflower, D.G. (2000). "Self-employment in OECD countries," Labour Economics 7, 471505 .

Blumberg, B. and G. Pfann (2015). "Roads leading to self-employment: comparing transgenerational entrepreneurs and self-made starts-ups," IZA DP 9155.

Bosma, N., van Praag, M., Thurik, R. and G. de Wit (2004). "The value of human and social capital investments for the business performance of start-ups," Small Business Economics $23,227-236$.

Breiman, L. (1996). "Bagging predictors". Machine Learning 24 (2): 123-140.

Breiman, L. (2001a). "Random Forests". Machine Learning 45 (1): 5-32.

Breiman, L. (2001b). "Statistical modeling: The two cultures (with comments and re-joinder by the author)," Statistical Science 16, 199-231.

Cooper, A.C. and X. Yin (2005). "Entrepreneurial networks," in Hitt, M.A. and R.D. Ireland (eds.), The Blackwell encyclopedia of management -entrepreneurship. Malden, MA: Blackwell, pp. 98-100.

Davidson, R., and J.G. MacKinnon,(2006). "Bootstrap methods in econometrics".

Davidsson, P. (1989). "Entrepreneurship -and after? A study of growth willingness in small firms," Journal of Business Venturing 4, 211-226.

Efron, B. (1979). "Bootstrap Methods: Another Look at the Jackknife". The Annals of Statistics 7, 1-26.

Efron, B. (1982). "The jackknife, the bootstrap, and other resampling plans," Society of Industrial and Applied Mathematics CBMS-NSF Monographs, 38.

Efron, B. And R. Tibshirani (1993). "An introduction to the bootstrap," Chapman and Hall.

Friedman, M. (1953). "The methodology of positive economics".

Friedman, J., Hastie, T., and R. Tibshirani. (2001). The elements of statistical learning (Vol. 1). Springer, Berlin: Springer series in statistics. 
Gilbert, B.A., McDougall, P.P. and D.B. Audretsch (2006). "New venture growth: A review and extension," Journal of Management 32, 926-950.

Gonçalves, S. and H. White (2005). "Bootstrap Standard Error Estimates for Linear Regression," Journal of the American Statistical Association 100, 970-979.

Ho, T.K. (1998). "The random subspace method for constructing decision forests," Pattern Analysis and Machine Intelligence, IEEE Transactions on 20, 838-844.

Holcomb, T.R., Ireland, R.D., Holmes, R.M. and M.A. Hitt (2009). "Architecture of entrepreneurial learning: exploring the link among heuristics, knowledge, and action," Entrepreneurship Theory and Practice 33, 167-192.

Horowitz, J.L. (1995). Advances in economics and econometrics: theory and applications, chapter 7: Bootstrap methods in econometrics: theory and numerical performance.

Horowitz, J.L. (2003). "The bootstrap in econometrics," Statistical Science 18, 211-218.

James, G., Witten, D., Hastie, T. And T. Tibshirani (2013). An introduction to Statistical Learning (Vol. 112). Springer, New York.

Jeong, J. and G.S. Maddala (1993). "A perspective on application of bootstrap methods in econometrics," Handbook of Statistics 11, 573-610.

Karlan, D. and M. Valdivia (2011). "Teaching entrepreneurship: Impact of business training on mincrofinance clients and institutions," Review of Economics and Statistics 93, 510-552.

Kelley, D. (2009). "Growth aspirations as a function of entrepreneurial motivations and perceptions," Babson Faculty Research Working Papers no. 49.

Kotsova, T. (1997). "Country institutional profiles concept and measurement," Academy of Management Proceedings 97, 180-184.

Kyrö, P. (2015). "The conceptual contribution of education to research on entrepreneurship education," Entrepreneurship and Regional Development, 1-20.

Levie, J. and E. Autio (2013). "Growth and growth intentions: A meta-analysis of existing evidence," Enterprise Research Centre, ERC White Papers no. 1.

Lundstrom, A. and L. Stevenson (2002). "On the road to entrepreneurship policy," Vol. 1 in the Entrepreneurship Policy for the Future series. Stockholm: Swedish Foundation for Small Business Research.

MacKinnon, J.G. (2002). "Bootstrap Inference in Econometrics," The Canadian Journal of Economics 35, 615-645.

MacKinnon, J.G. (2006). "Bootstrap Methods in Econometrics," The Economic Record 82, S2S18.

Minniti, M. (2005). "Entrepreneurship and network externalities," Journal of Economic Behaviour \& Organization 57, 1-27.

Minniti, M. and C. Nardone (2007). "Being in someone else's shoes: Gender and nascent entrepreneurship," Small Business Economics 28, 223-239.

Molina, J.A., Velilla, J. and R. Ortega (2015). "The decision to become an entrepreneur in Spain: The role of the household financial situation," MPRA Paper no. 68101.

Murphy, K.M. and R.H: Topel (2002). "Estimation and Inference in Two-Step Econometric Models," Journal of Business and Economic Statistics 20, 88-97.

Nagler, P. and W. Naudé (2014). "Non-farm enterprises in rural Africa: New empirical evidence," Policy Research Working Paper no. 7066. Washington DC: The World Bank.

Naudé, W. (2016). "Is European Entrepreneurship in Crisis?," IZA DP no. 9817. 
Pagan, A. (1984). "Econometric issues in the analysis of regressions with generated regressors," International Economic Review, 221-247.

Schott, T. and T. Bager (2004). "Growth expectations by entrepreneurs in nascent firms, baby business and mature firms," in T. Bager and M. Hancock (eds.), The growth of Danish firms (Part 2 of the Global Entrepreneurship Monitor). Copenhagen, DK: Borsens Forlag, pp. 219-230.

Schumpeter, A. (1934). The Theory of Economic Development. Cambridge, MA: Harvard University Press.

Terjesen, S. and L. Szerb (2008). "Dice thrown from the beginning? An empirical investigation of firm level growth expectations," Estudios de Economía 35, 157-178.

Vinod, H.D. (1993). "Bootstrap methods: Applications in econometrics," Handbook of Statistics $11,629-661$. 
Table 1. Macro-economic factors - GEM 2014 NES National

\begin{aligned} \hline TEA & Total (Early-Stage) Entrepreneurial Activity index \\ Country & $\begin{array}{l}\text { Country name/code } \\ \text { Eelonging to EU }\end{array} \\$ OECD & $\begin{array}{l}\text { Belonging to the OECD } \\ \text { Group A }\end{array} \\$ A.1 & In my country, there is sufficient equity funding available for new and growing firms \\ A.2 & In my country, there is sufficient debt funding available for new and growing firms \\ A.3 & In my country, there are sufficient government subsidies available for new and growing firms \\ A.4 & In my country, there is sufficient funding available from private individuals (other than founders) \\ A.5 new and growing firms & In my country, there is sufficient venture capitalist funding available for new and growing firms) \\ A.6 & In my country, there is sufficient funding available through initial public offerings (IPOs) for \\ & new and growing firms \end{aligned}

Group B1 (Government concrete policies, priority, and support)

B1.1 In my country, Government policies (e g, public procurement) consistently favor new firms

B1.2 In my country, the support for new and growing firms is a high priority for policy at the national government level

B1.3 In my country, the support for new and growing firms is a high priority for policy at the local government level

B1.4 In my country, new firms can get most of the required permits and licenses in about a week

Mean S. Dev

$13.084 \quad \frac{\text { Dev }}{8.156}$

$0.333-0.474$

$0.420 \quad 0.497$

$2.535 \quad 0.397$

$2.595 \quad 0.499$

$2.684 \quad 0.434$

$2.715 \quad 0.588$

$2.574 \quad 0.388$

$2.460 \quad 0.480$

$2.286 \quad 0.590$

$2.617 \quad 0.427$

$2.237 \quad 0.407$

$2.875 \quad 0.532$

$2.716 \quad 0.492$

$2.227 \quad 0.677$

Group B2 (Government policies bureaucracy, taxes)

$\begin{array}{ll}2.462 & 0.569\end{array}$

B2.5 In my country, the amount of taxes is NOT a burden for new and growing firms

$2.455 \quad 0.616$

B2.6 In my country, taxes and other government regulations are applied to new and growing firms in a predictable and consistent way

$2.767 \quad 0.642$

B2.7 In my country, coping with government bureaucracy, regulations, and licensing requirements it is not unduly difficult for new and growing firms

$2.432 \quad 0.611$

$2.679 \quad 0.438$

$\begin{aligned} & \text { Group C } \text { (Government programs) } \\ & \text { C.1 In my country, a wide range of government assistance for new and growing firms can be }\end{aligned}$ obtained through contact with a single agency

C.2 In my country, science parks and business incubators provide effective support for new and growing firms

$2.423 \quad 0.598$

$3.106 \quad 0.571$

C.3 In my country, there are an adequate number of government programs for new and growing businesses

2.896

0.559

C.4 In my country, the people working for government agencies are competent and effective in supporting new and growing firms

C.5 In my country, almost anyone who needs help from a government program for a new or growing business can find what they need

C.6 In my country, Government programs aimed at supporting new and growing firms are effective

$2.706 \quad 0.428$

$2.448 \quad 0.447$

$2.625 \quad 0.414$

Group D1 (Entrepreneurial level of education at Primary and Secondary)

$2.091 \quad 0.398$

D1.1 In my country, teaching in primary and secondary education encourages creativity, selfsufficiency, and personal initiative

D1.2 In my country, teaching in primary and secondary education provides adequate instruction in market economic principles

D1.3 In my country, teaching in primary and secondary education provides adequate attention to entrepreneurship and new firm creation

$2.270 \quad 0.486$

$2.091 \quad 0.410$

$1.944 \quad 0.390$

Group D2 (Entrepreneurial level of education at vocational, professional, College and $2.891 \quad 0.306$ University)

D2.4 In my country, Colleges and universities provide good and adequate preparation for starting up and growing new firms

D2.5 In my country, the level of business and management education provide good and adequate preparation for starting up and growing new firms

D2.6 In my country, the vocational, professional, and continuing education systems provide good and adequate preparation for starting up and growing new firms 
universities and public research centers to new and growing firms

E.2 In my country, new and growing firms have just as much access to new research and technology as large, established firms

E.3 In my country, new and growing firms can afford the latest technology

E.4 In my country, there are adequate government subsidies for new and growing firms to acquire new technology

E.5 In my country, the science and technology base efficiently supports the creation of world-class new technology-based ventures in at least one area

E.6 In my country, there is good support available for engineers and scientists to have their ideas commercialized through new and growing firms

F.1 In my country, there are enough subcontractors, suppliers, and consultants to support new and growing firms

F.2 In my country, new and growing firms can afford the cost of using subcontractors, suppliers, and consultants

F.3 In my country, it is easy for new and growing firms to get good subcontractors, suppliers, and consultants

F.4 In my country, it is easy for new and growing firms to get good, professional legal and accounting services

F.5 In my country, it is easy for new and growing firms to get good banking services (checking accounts, foreign exchange transactions, letters of credit, and the like)

Group G1 (Internal market dynamics)

G1.1 In my country, the markets for consumer goods and services change dramatically from year to year

G1.2 In my country, the markets for business-to-business goods and services change dramatically from year to year

Group G2 (Internal market burdens)

G2.3 In my country, new and growing firms can easily enter new markets

$2.730 \quad 0.331$

G2.4 In my country, the new and growing firms can afford the cost of market entry

G2.5 In my country, new and growing firms can enter markets without being unfairly blocked by established firms

G2.6 In my country, the anti-trust legislation is effective and well enforced

Group H (Physical infrastructures and services access)

H.1 In my country, the physical infrastructure (roads, utilities, communications, waste disposal) provides good support for new and growing firms

H.2 In my country, it is not too expensive for a new or growing firm to get good access to communications (phone, Internet, etc)

H.3 In my country, a new or growing firm can get good access to communications (telephone, internet, etc) in about a week

H.4 In my country, new and growing firms can afford the cost of basic utilities (gas, water, electricity, sewer)

H.5 In my country, new or growing firms can get good access to utilities (gas, water, electricity, sewer) in about a month

Group I (Cultural, social norms and society support)

I.1 In my country, the national culture is highly supportive of individual success achieved through own personal efforts

I.2 In my country, the national culture emphasizes self-sufficiency, autonomy, and personal initiative

I.3 In my country, the national culture encourages entrepreneurial risk-taking

I.4 In my country, the national culture encourages creativity and innovativeness

I.5 In my country, the national culture emphasizes the responsibility that the individual (rather than 
Table 2. Summary statistics - GEM 2014 Global Individual

\begin{tabular}{|c|c|c|c|c|c|}
\hline & \multicolumn{2}{|c|}{ OECD } & \multicolumn{2}{|c|}{ Non-OECD } & \multirow[b]{2}{*}{$p$-value } \\
\hline Variables & $\underline{\text { Mean }}$ & $\underline{\text { S. Dev. }}$. & Mean & $\underline{\text { S. Dev. }}$ & \\
\hline Contributes to TEA index & 0.0813 & 0.2733 & 0.1621 & 0.3686 & $(<0.01)$ \\
\hline Age & 43.043 & 14.700 & 38.158 & 13.656 & $(<0.01)$ \\
\hline Being male & 0.4895 & 0.4998 & 0.4788 & 0.4995 & $(<0.01)$ \\
\hline Family number & 3.2051 & 1.6032 & 4.1119 & 2.1926 & $(<0.01)$ \\
\hline Number of children & 0.8238 & 1.3321 & 0.2612 & 0.8671 & $(<0.01)$ \\
\hline Basic education & 0.1858 & 0.3889 & 0.0499 & 0.2178 & $(<0.01)$ \\
\hline Secondary education & 0.5879 & 0.4922 & 0.6248 & 0.4841 & $(<0.01)$ \\
\hline University education & 0.1639 & 0.3702 & 0.2929 & 0.4551 & $(<0.01)$ \\
\hline Consider to have skills to be entrepreneur & 0.4396 & 0.4963 & 0.5482 & 0.4976 & $(<0.01)$ \\
\hline Low level of income & 0.2554 & 0.4361 & 0.3021 & 0.4592 & $(<0.01)$ \\
\hline Middle level of income & 0.2503 & 0.4332 & 0.2682 & 0.4432 & $(<0.01)$ \\
\hline High level of income & 0.2470 & 0.4313 & 0.2672 & 0.4425 & $(<0.01)$ \\
\hline Being employed & 0.5460 & 0.4978 & 0.3944 & 0.4887 & $(<0.01)$ \\
\hline Being self-employed & 0.1411 & 0.3471 & 0.2895 & 0.4887 & $(<0.01)$ \\
\hline Being unemployed & 0.1170 & 0.3214 & 0.1353 & 0.3420 & $(<0.01)$ \\
\hline Being an active unemployed & 0.1166 & 0.3209 & 0.1349 & 0.3416 & $(<0.01)$ \\
\hline Being retired & 0.1336 & 0.3402 & 0.0885 & 0.2688 & $(<0.01)$ \\
\hline Being a student & 0.1024 & 0.3019 & 0.1154 & 0.3195 & $(<0.01)$ \\
\hline Being a homemaker & 0.1338 & 0.3404 & 0.2510 & 0.4335 & $(<0.01)$ \\
\hline Being a businessman & 0.1069 & 0.3088 & 0.1927 & 0.3944 & $(<0.01)$ \\
\hline Desire to be a businessman & 0.4396 & 0.4963 & 0.6618 & 0.4730 & $(<0.01)$ \\
\hline Desire to be entrepreneur in the future & 0.1393 & 0.3463 & 0.3200 & 0.4665 & $(<0.01)$ \\
\hline Know someone who is an entrepreneur & 0.3916 & 0.8981 & 0.5032 & 0.9155 & $(<0.01)$ \\
\hline Consider to have opportunities to entr. & 0.2684 & 0.4431 & 0.4364 & 0.4959 & $(<0.01)$ \\
\hline Have a good perception of entrepreneur & 0.2682 & 0.4430 & 0.4406 & 0.4964 & $(<0.01)$ \\
\hline Consider that culture support entrep. & 0.4281 & 0.4948 & 0.6671 & 0.4712 & $(<0.01)$ \\
\hline Have fear of failure & 0.4385 & 0.4962 & 0.3543 & 0.4783 & $(<0.01)$ \\
\hline Desire for social equity & 0.4931 & 0.4999 & 0.5670 & 0.4954 & $(<0.01)$ \\
\hline Respect for success & 0.5412 & 0.4983 & 0.6673 & 0.4711 & $(<0.01)$ \\
\hline Media promotes successful businessmen & 0.4155 & 0.4928 & 0.6420 & 0.4794 & $(<0.01)$ \\
\hline Have helped other entrepreneurs & 0.0707 & 0.2564 & 0.1262 & 0.3321 & $(<0.01)$ \\
\hline Can offer a new product & 0.0637 & 0.2442 & 0.0950 & 0.2932 & $(<0.01)$ \\
\hline Can offer a new technology & 0.0344 & 0.1823 & 0.0750 & 0.2634 & $(<0.01)$ \\
\hline Technological level of the sector working & 0.0096 & 0.0975 & 0.0047 & 0.0685 & $(0.0651)$ \\
\hline Search for new markets & 0.1503 & 0.3574 & 0.2619 & 0.4397 & $(<0.01)$ \\
\hline Search for new customers & 0.0031 & 0.0562 & 0.0158 & 0.1248 & $(<0.01)$ \\
\hline Have ended a business recently & 0.0288 & 0.1674 & 0.0609 & 0.2392 & $(<0.01)$ \\
\hline Have invested in other business & 0.0487 & 0.2155 & 0.0599 & 0.2374 & $(<0.01)$ \\
\hline Belong to a developing country & - & - & 0.7721 & 0.4194 & $(<0.01)$ \\
\hline Belong to a not-developed country & - & - & 0.1680 & 0.3739 & $(<0.01)$ \\
\hline Have an ideal life & 0.4969 & 0.4999 & 0.5288 & 0.4991 & $(<0.01)$ \\
\hline Have good conditions of life & 0.4986 & 0.5000 & 0.5022 & 0.4999 & $(0.1153)$ \\
\hline Be satisfied with life & 0.6782 & 0.4671 & 0.6177 & 0.4859 & $(<0.01)$ \\
\hline Obtain important things in life & 0.6028 & 0.4893 & 0.5173 & 0.4997 & $(<0.01)$ \\
\hline Satisfied with life decisions & 0.4409 & 0.4965 & 0.4257 & 0.4944 & $(<0.01)$ \\
\hline Have freedom at work & 0.3452 & 0.4754 & 0.3307 & 0.4704 & $(<0.01)$ \\
\hline Like current job & 0.8932 & 0.3088 & 0.8849 & 0.3201 & $(<0.01)$ \\
\hline Have a no--stressful job & 0.6852 & 0.4644 & 0.7770 & 0.4162 & $(<0.01)$ \\
\hline Satisfied with current job & 0.3788 & 0.4851 & 0.3018 & 0.4590 & $(<0.01)$ \\
\hline Satisfied with current income & 0.2322 & 0.4222 & 0.2226 & 0.4160 & $(<0.01)$ \\
\hline Enough time for leisure & 0.1947 & 0.3959 & 0.2965 & 0.4567 & $(<0.01)$ \\
\hline Enough time for family activities & 0.2007 & 0.4005 & 0.3097 & 0.4623 & $(<0.01)$ \\
\hline Enough time for housework activities & 0.2187 & 0.4133 & 0.3165 & 0.4651 & $(<0.01)$ \\
\hline Being an immigrant & 0.0487 & 0.2153 & 0.0113 & 0.1060 & $(<0.01)$ \\
\hline
\end{tabular}

Note: the sample (GEM 2014 Global Individual level) is restricted to individuals who reported age. Kruskal-Wallis $p$-values for the comparison of the variables among belonging or not belonging to the OECD in parenthesis. Age is measured in years. The rest of the variables are dummies, taking value 1 if agree or 0 if not agree. 
Table 3. Final logit model

\begin{tabular}{|c|c|c|c|c|}
\hline VARIABLES & Estimate & Std. Error & $t$-ratio & $p$-value \\
\hline Age & $-0.045 * * *$ & 0.001 & -39.204 & $(<0.001)$ \\
\hline Being a businessman & $-1.506 * * *$ & 0.043 & -34.746 & $(<0.001)$ \\
\hline Have ended a business recently & $0.134^{*}$ & 0.043 & 2.471 & $(0.013)$ \\
\hline Constant & -20.077 & 72.700 & -0.276 & $(0.782)$ \\
\hline
\end{tabular}

Note: the sample (GEM 2014 Global Individual) is restricted to individuals who reported age. Dependent variable is the dummy "contributes to TEA". 
Figure 1. Results for GEM 2014 NES National Level data

Panel A

Disagregated Variables

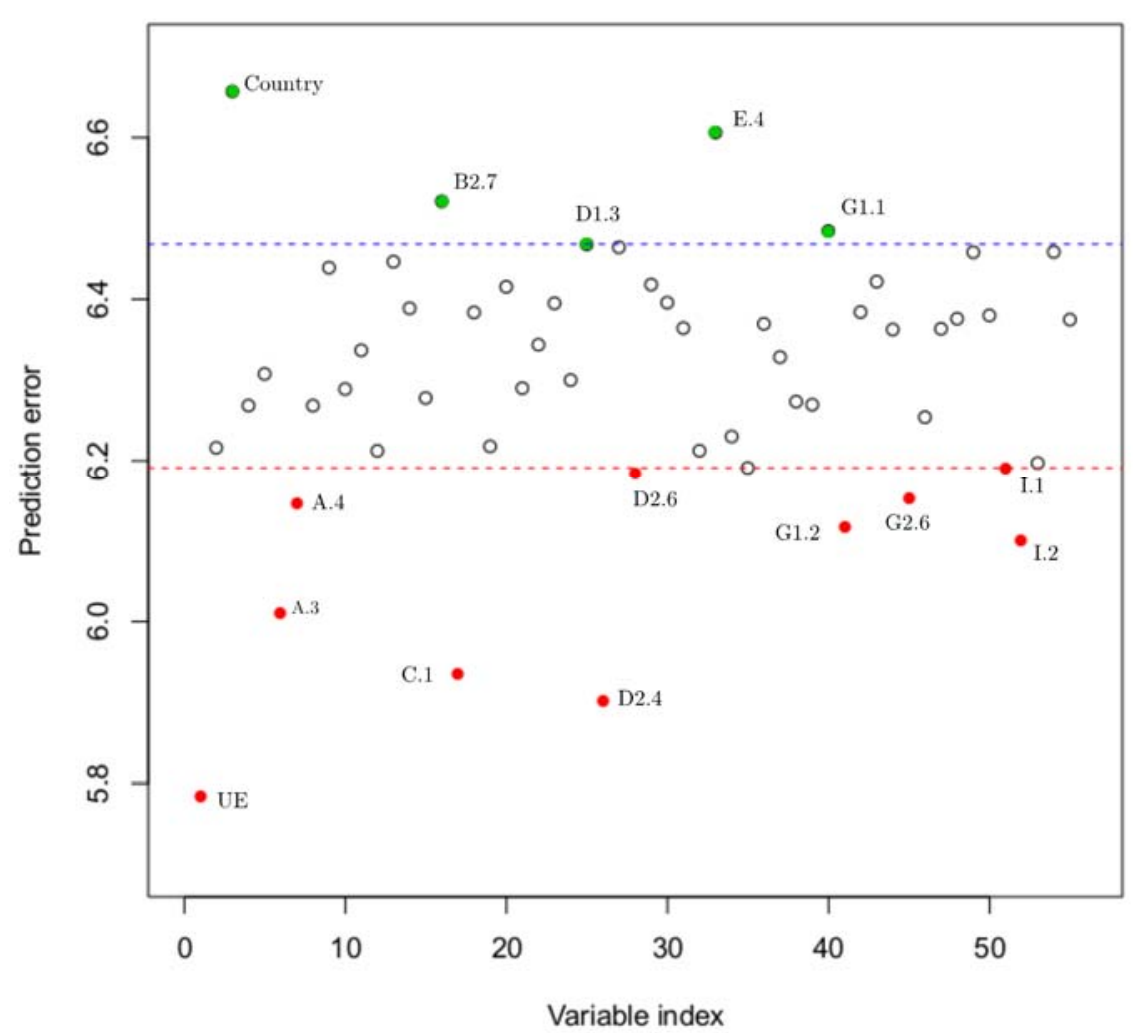

Panel B

Principal components

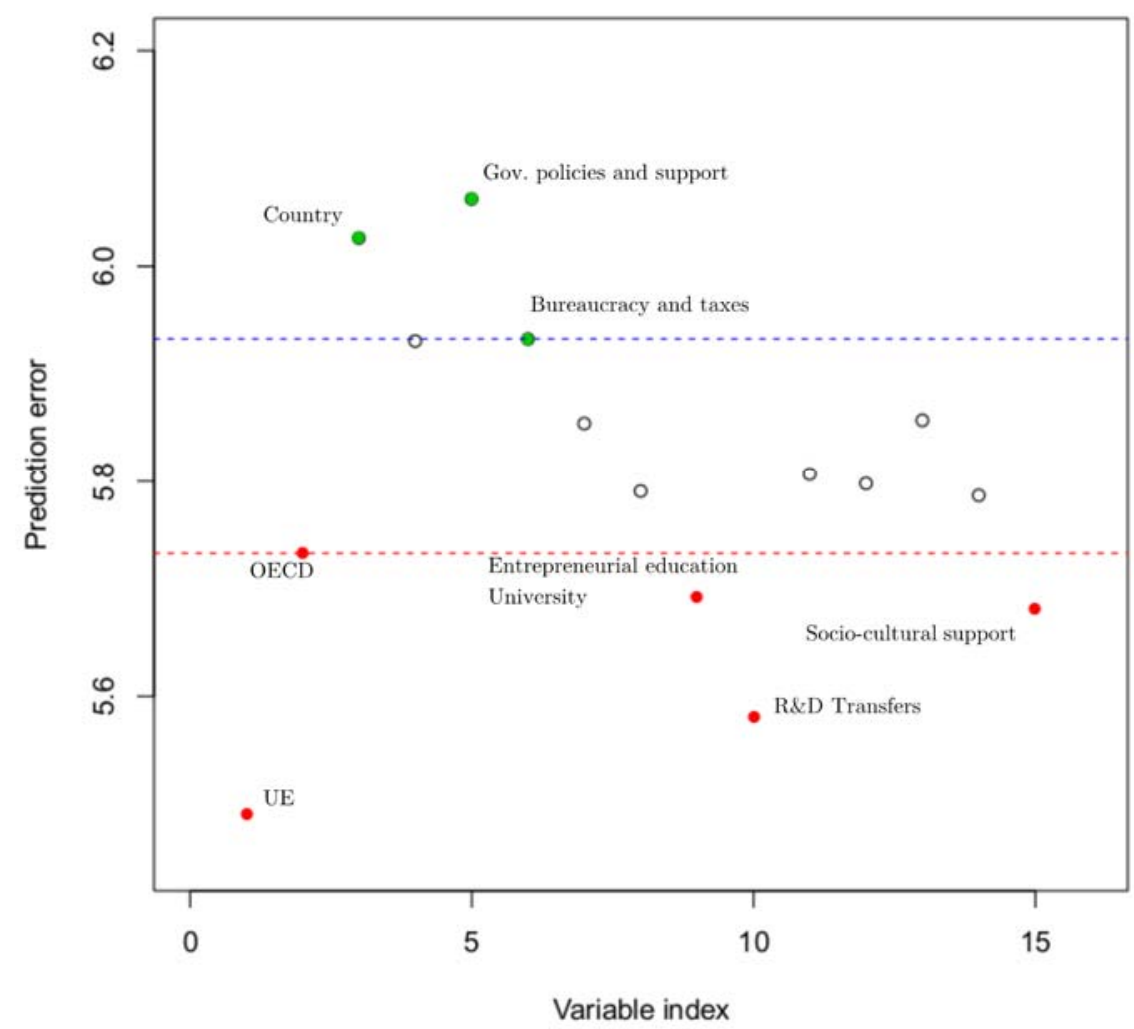

Note: the sample is GEM 2014 NES National. Dependent variable is the "TEA Index". In Panel A we use the disaggregated variables, while in Panel B we use aggregated principal component factors (variable indices are taken ordered from Table 1). We compute 500 iterations bootstrap of linear regressions, following the method described in Sections 2. 
Figure 2. Forward stepwise for principal factors analysis of GEM 2014 NES National Level data

Panel A

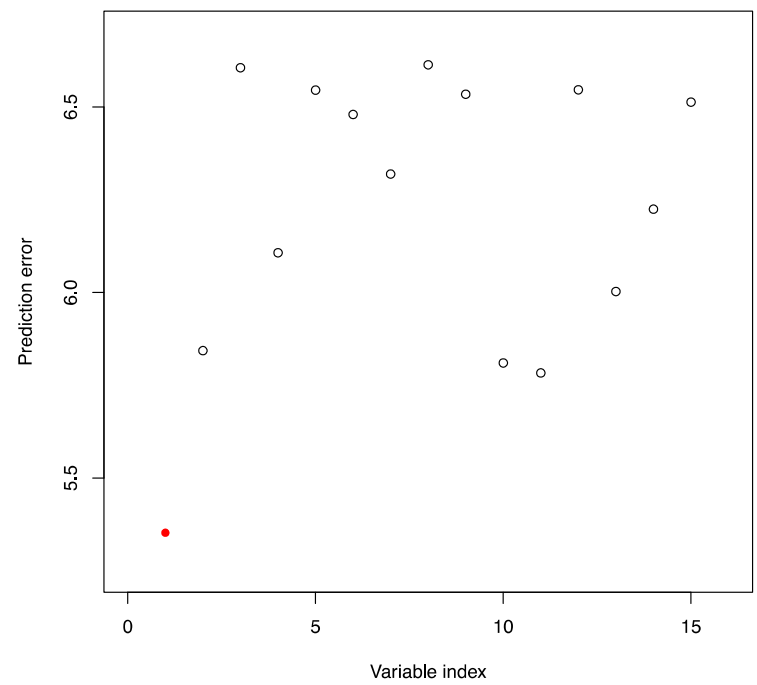

Panel C

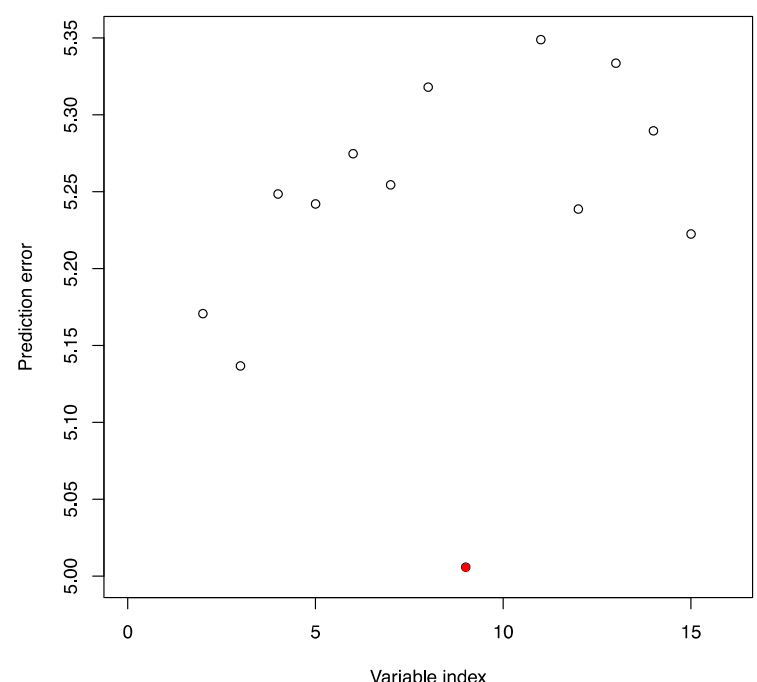

Panel B

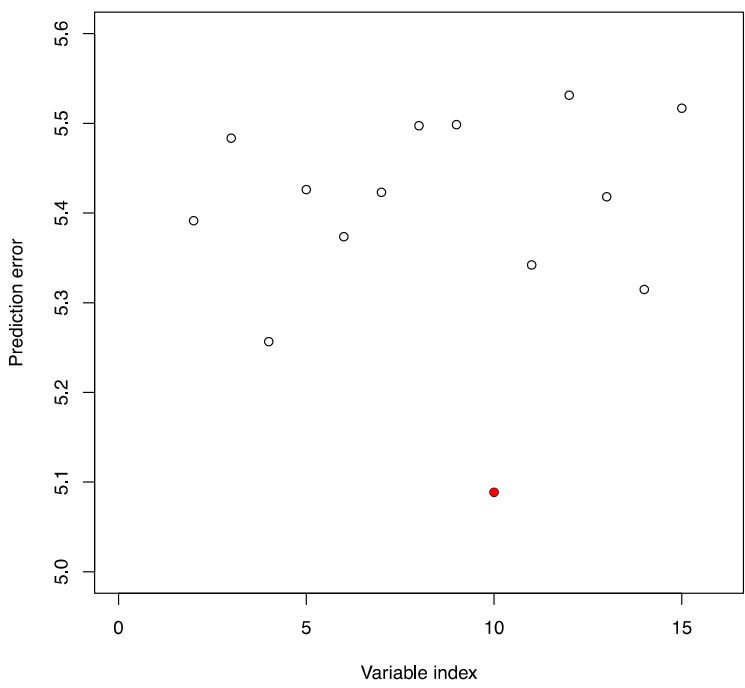

Panel D

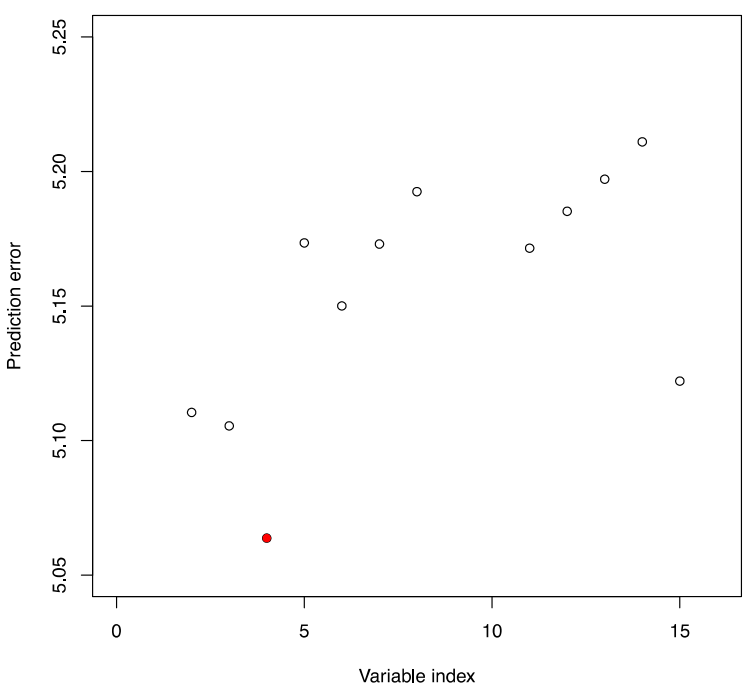

Note: the sample (GEM 2014 NES National) is restricted to individuals who reported age. Dependent variable is "TEA index". Regressors are aggregated principal component factors (see Table 1). In Panel A, we show results of 2,000 iterations bootstrap of linear regressions with a single regressor in each iteration. The variable with the highest predictive power is "EU" (index 1). In Panel B, we repeat the process selecting "EU" and another variable in each iteration. The variable with the highest predictive power is now the R\&D transfers level (index 10). Repeating analogously, in Panel C, the variable with the highest predictive power is now the level of vocational, professional, College, and University entrepreneurial education (index 9). Finally, in panel $\mathrm{D}$, the selected variable is the financial environment (index 4). Prediction errors (mean absolute errors) decrease in Panel A, B and $\mathrm{C}$, but increase in Panel D. 
Figure 3. Forward stepwise for all individual variables of GEM 2014 NES National Level data

Panel A

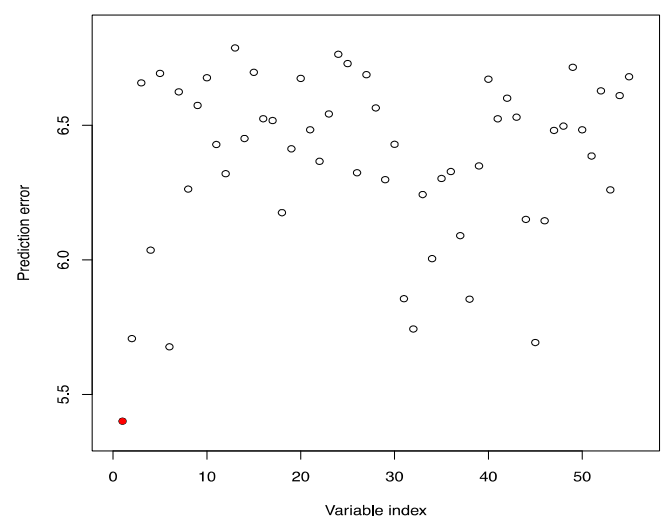

Panel C

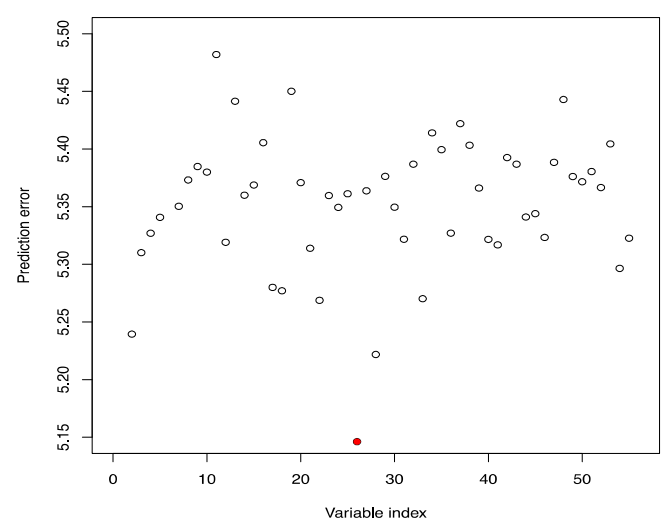

Panel E

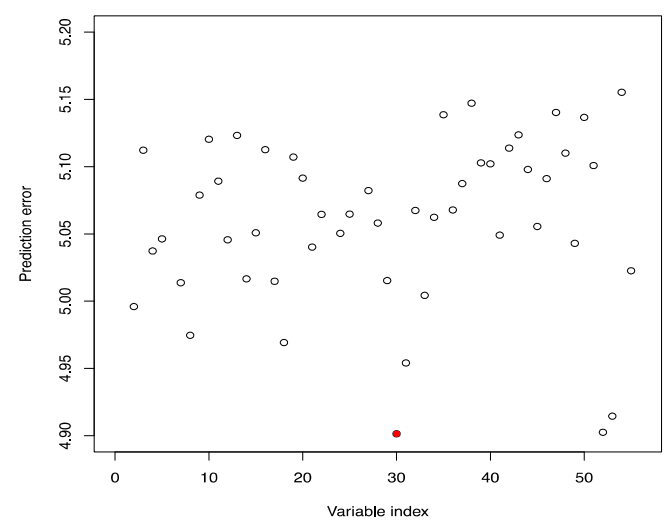

Panel B

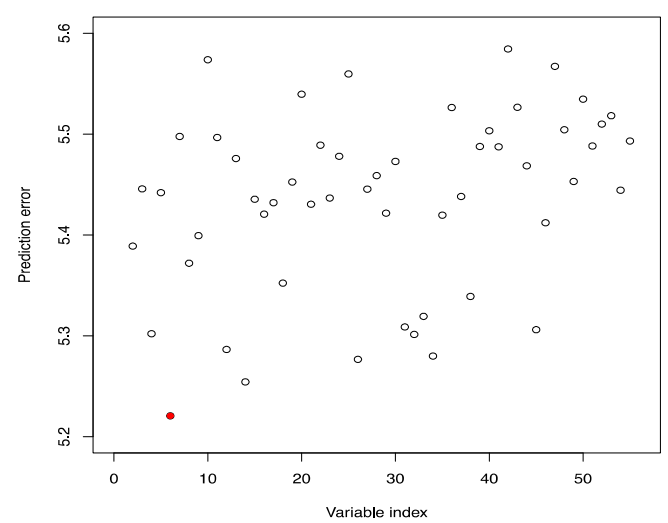

Panel D

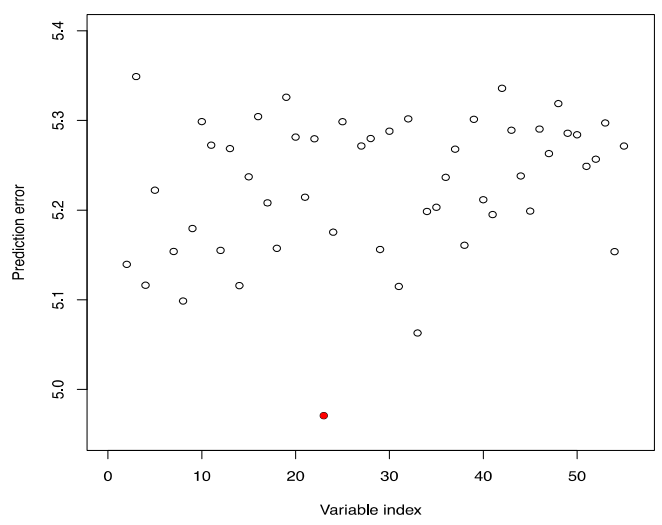

Panel F

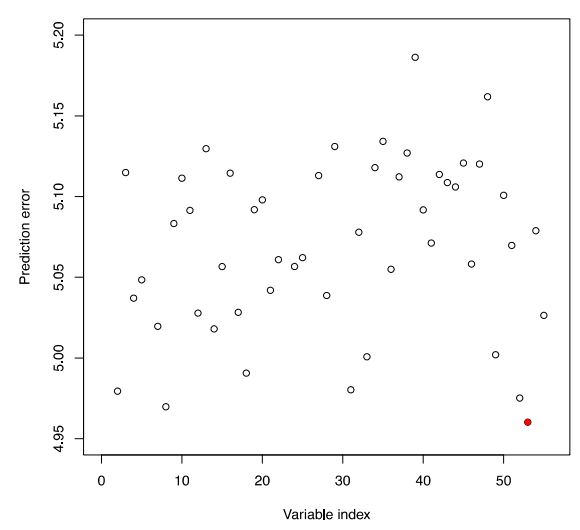

Note: the sample (GEM 2014 NES National) is restricted to individuals who reported age. Dependent variable is "TEA index". Regressors are disaggregated variables (see Table 1). In Panel A, we show results of 2,000 iterations bootstrap of linear regressions with a single regressor in each iteration. The variable with the highest predictive power is "EU" (index 1). In Panel B, we repeat the process selecting "EU" and another variable in each iteration. The variable with the highest predictive power is now government subsidies for new and growing firms (index 6). Repeating analogously, in Panel C, the variable with the highest predictive power is now entrepreneurial preparation at University (index 26); in Panel D, creativity, self-sufficiency, and initiative at primary/secondary school (index 23); and in Panel E, equity of access to technology by new and growing forms (index 30). Finally, we repeat the process in panel $\mathrm{F}$ and the selected variable is now the culture of encouragement of entrepreneurial risk-taking (index 53). Prediction errors (mean absolute errors) decrease in Panel A, B, C, D and E but increase in Panel F. 
Figure 4. Application of the algorithm to classification problems with Regression Model GEM 2014 Individual Level data

500 R.log

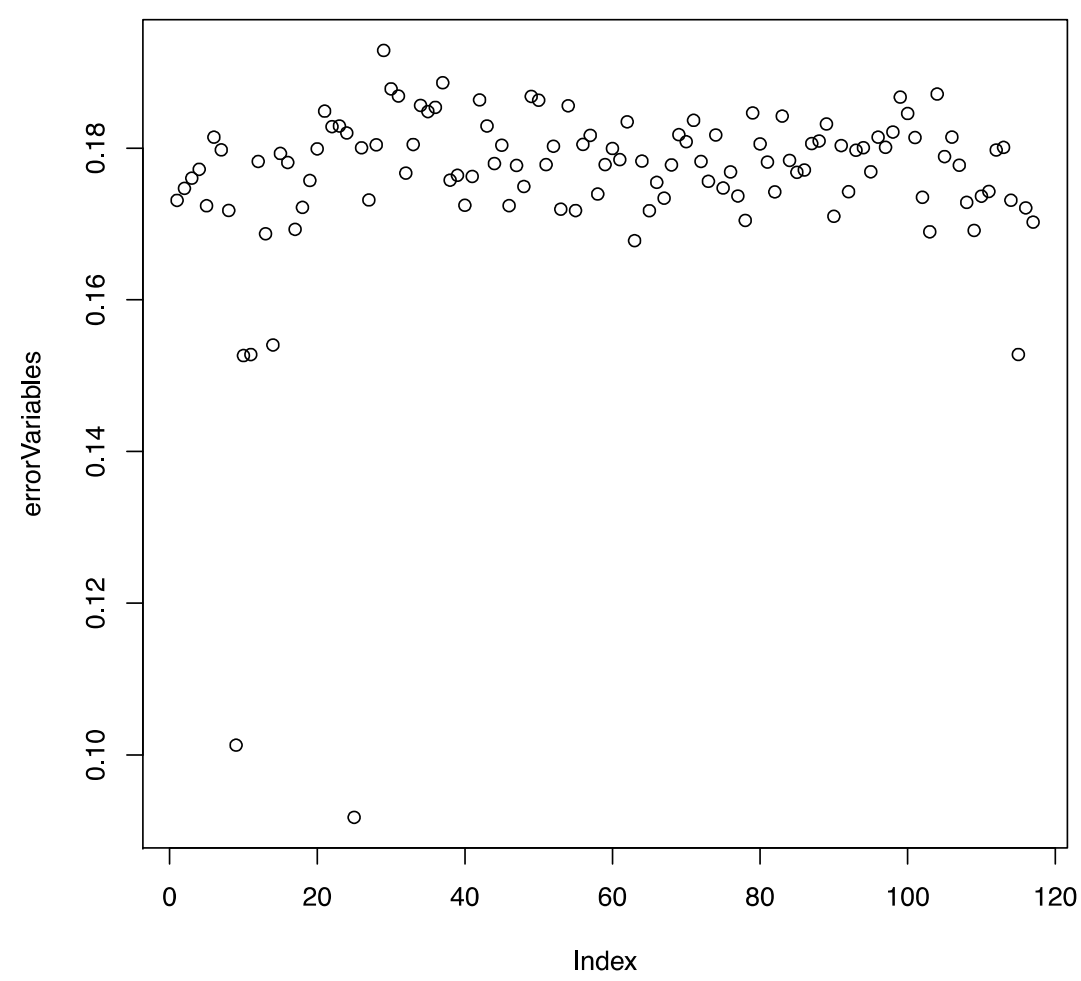

LDA Model

500 LDA

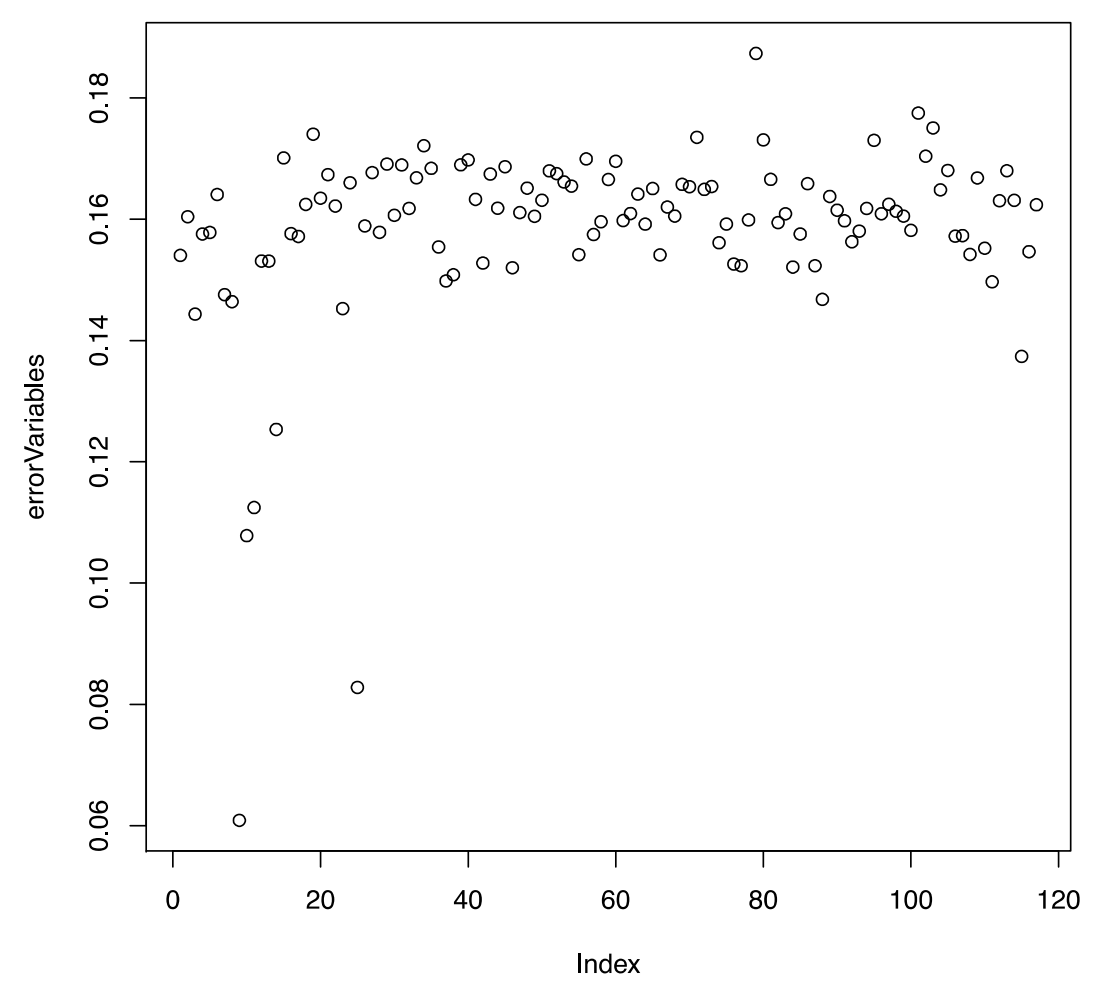

Note: the sample (GEM 2014 Global Individual) is restricted to individuals who reported age. Dependent variable is the dummy "contributes to TEA". Panel A shows results for a logistic regression problem, and Panel B for a Linear Discriminant Analysis. We compute 500 iterations bootstrap of logistic and LDA classification problems, following the method described in Sections 2 . 
Figure 5. Forward stepwise for classification problems with GEM 2014 Individual Level data

Regression Model

nlter=10, var=10

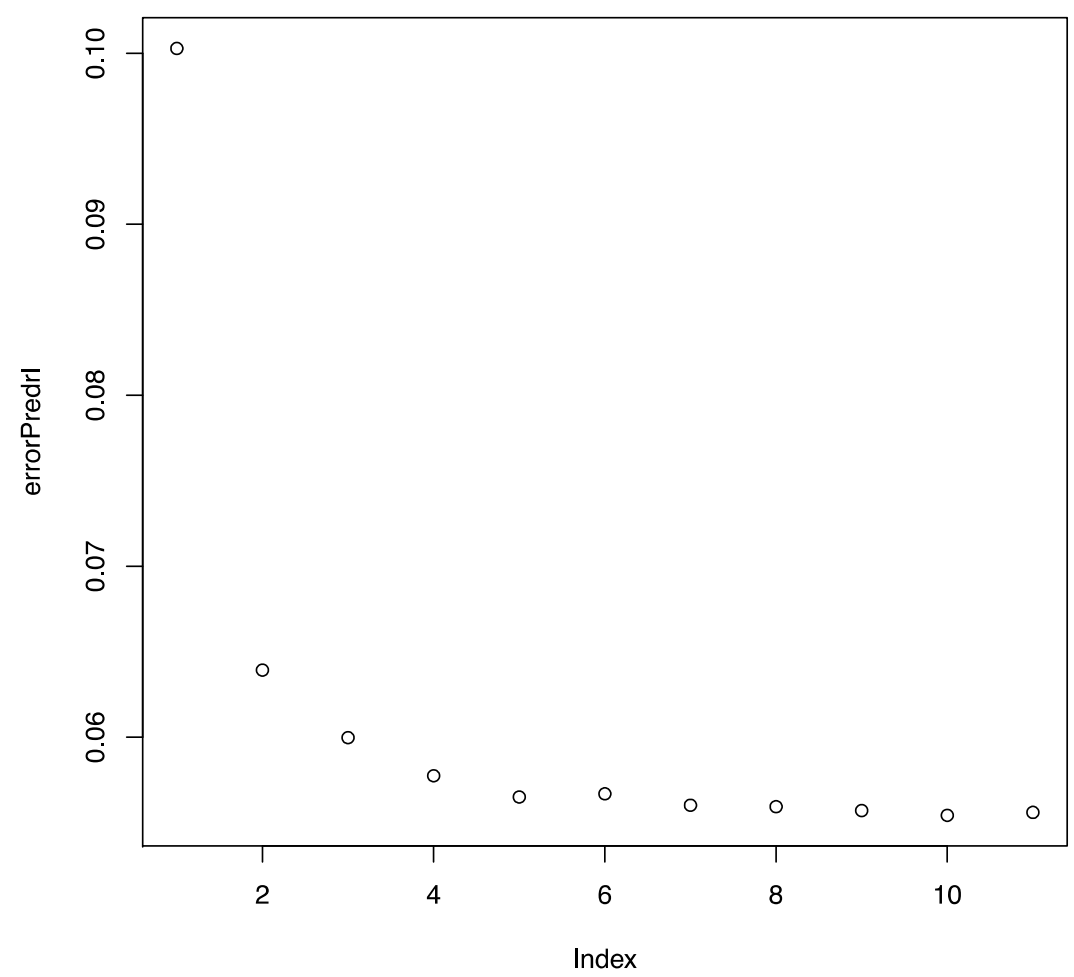

LDA Model

nlter $=10$, var=10

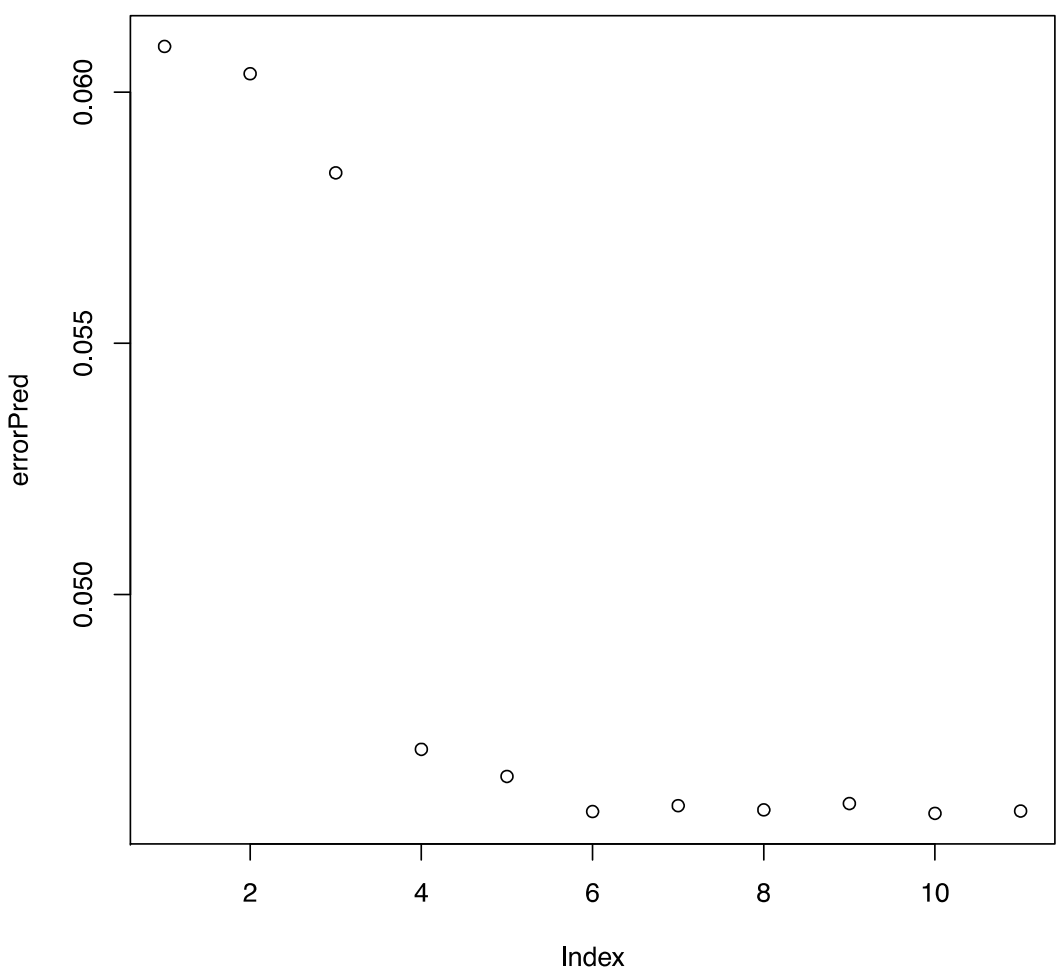

Note: the sample (GEM 2014 Global Individual) is restricted to individuals who reported age. Dependent variable is the dummy "contributes to TEA". In Panel A, we show results of 10 iterations bootstrap of logistic regressions following a forward stepwise procedure. In Panel B, we repeat the process for a LDA. 


\section{Appendix: R-code of the algorithmic approach}

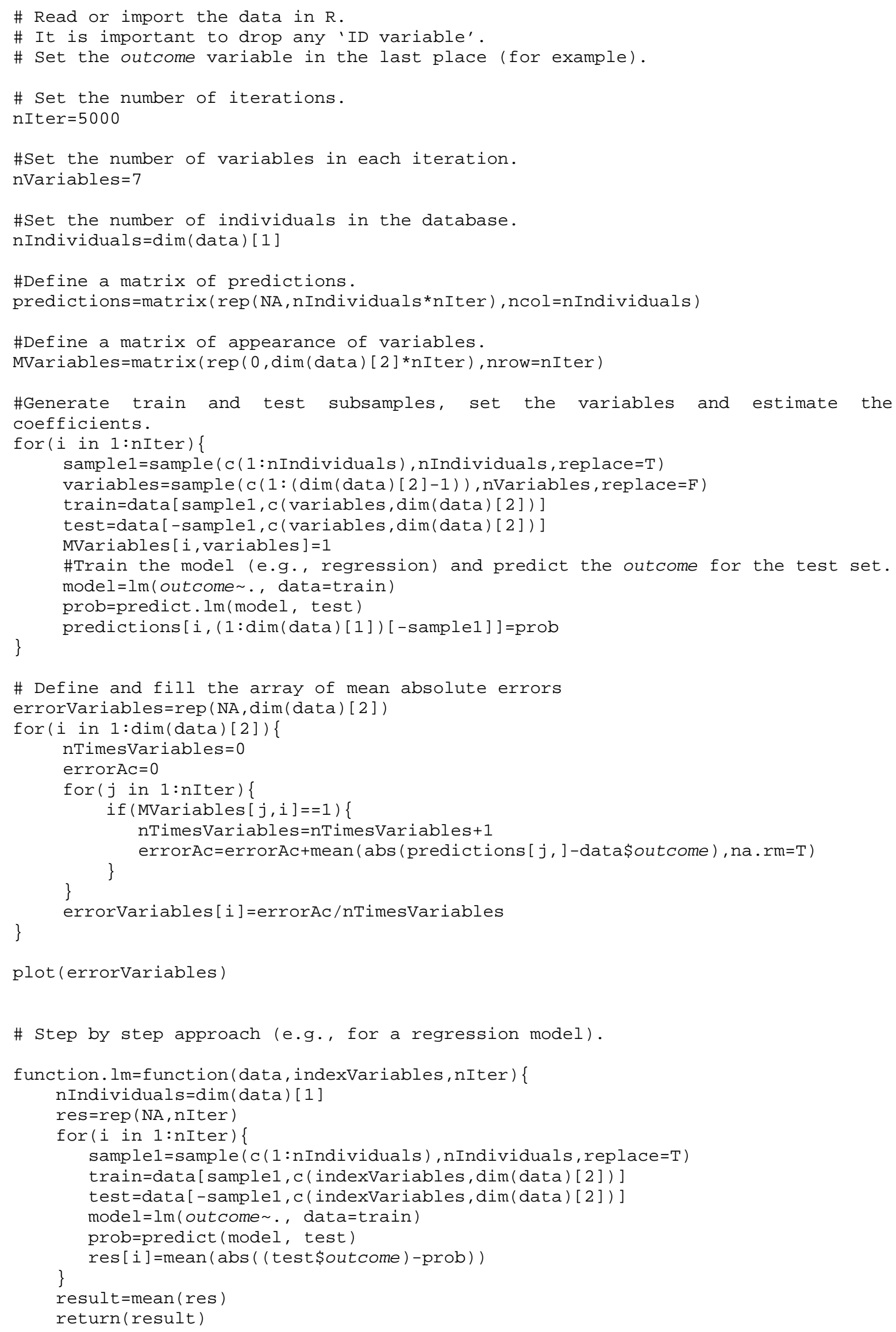


\}

errors. $1 \mathrm{~m}=\mathrm{rep}(\mathrm{NA}$, number of features)

for ( $j$ in 1:number of features) \{

errors. $\operatorname{lm}[j]=$ function. $\operatorname{lm}(\operatorname{data}, c(j), 2000)$

$\operatorname{print}(j)$

plot (errors.lm)

\#Repeat excluding the variable with the lowest 'error.lm' until required. 